OPEN ACCESS

Edited by: Jingqiang Tan,

Central South University, China

Reviewed by:

Shouzhi Hu,

China University of Geosciences

Wuhan, China

Jiwei Li,

Institute of Deep-Sea Science and

Engineering (CAS), China

${ }^{*}$ Correspondence:

Wei Yuan

qujiingyw@qq.com

Specialty section:

This article was submitted to

Geochemistry,

a section of the journal

Frontiers in Earth Science

Received: 30 September 2021

Accepted: 25 January 2022

Published: 14 February 2022

Citation:

Yuan W, Liu G, Zhou X and Bulseco A (2022) Linking Hydrothermal Activity With Organic Matter Accumulation in the Chang 7 Black Shale of Yanchang

Formation, Ordos Basin, China.

Front. Earth Sci. 10:786634.

doi: 10.3389/feart.2022.786634

\section{Linking Hydrothermal Activity With Organic Matter Accumulation in the Chang 7 Black Shale of Yanchang Formation, Ordos Basin, China}

\author{
Wei Yuan ${ }^{1 *}$, Guangdi Liu ${ }^{2}$, Xiaoxing Zhou ${ }^{3}$ and Ashley Bulseco ${ }^{4}$ \\ ${ }^{1}$ College of Geosciences, Northeast Petroleum University, Daqing, China, ${ }^{2}$ College of Geosciences, China University of \\ Petroleum, Beijing, China, ${ }^{3}$ Department of Graduate Affairs, Northeast Petroleum University, Daqing, China, ${ }^{4}$ Marine Science \\ Discipline, Eckerd College, St. Petersburg, FL, United States
}

The Chang 7 black shale in the Upper Triassic Yanchang Formation is the principal source rock of Mesozoic oil-bearing system in the southwest Ordos Basin, containing high abundances of organic matter and hydrocarbon potential. Our study discusses the role of lake-bottom hydrothermal activities in the enrichment of organic matter during the deposition of the Chang 7 black shale. A large number of basement faults developed in the interior and margin of the Ordos Basin, which provided channels for the upwelling of deep hydrothermal fluids. Moreover, the strong tectonic activities during the Chang 7 sedimentary period provided dynamic conditions for the activation of the faults and the upwelling of hydrothermal fluids. The occurrence of hydrothermal activities in the Chang 7 sedimentary period is proved by the evidences of mineralogy petrology, stable isotopes, major, and trace elements in the black shale. Abundant nutrients that were transported from the lake-bottom hydrothermal fluids into lake water promoted the lacustrine surface primary productivity, and then increased the supply of sedimentary organic matter. At the same time, the degradation of a large number of organic matters increased consumption of oxygen in the water column, resulting in the formation of bottom-water anoxic environments. The accumulation of organic matter in sediments was controlled by the lake-bottom hydrothermal activities by the means of increasing the lacustrine surface paleoproductivity and promoting the formation of anoxic environments.

Keywords: tectonic activities, carbon and oxygen isotopes, major and trace elements, nutrient elements, paleoproductivity, redox conditions

\section{INTRODUCTION}

Hydrothermal activity refers to the deposition, crystallization, metasomatism, alteration, and filling of high temperature fluids that rise from the deep earth to the surface. These fluids generally contain ore-forming materials that are important for mineralization, and result in high-grade and large-scale mineral deposits along hydrothermal sedimentary areas (Zhang et al., 2010; Jia et al., 2016). Hydrothermal activity occurs in various environments, including mid-ocean ridges, oceanic inland arcs, back-arc basins, faulted basins, continental margins, and even relatively stable depression lake basins (Rona et al., 1975; Boström et al., 1979; Crerar et al., 1982; Marchig et al., 1982; Adachi et al., 1986; Yamamoto, 1987; Rona, 1988; Cronan et al., 1995; Hodkinson 


\begin{tabular}{|c|c|c|c|c|}
\hline System & Basin / Aare & Formation & Facies & Lithology \\
\hline Paleogene & Bohai Bay Basin & Shahejie & Lacustrine & Limestone \\
\hline Cretaceous & Jiuquan Basin & Xiagou & Lacustrine & Dolomite \\
\hline Triassic & Ordos Basin & Yanchang & Lacustrine & Silicolite \\
\hline \multirow{7}{*}{$\begin{array}{c}\stackrel{\Xi}{\Xi} \\
\stackrel{\Xi}{0} \\
\stackrel{0}{0}\end{array}$} & Junggar Basin & Lucaogou & Transitional & Silicolite \\
\hline & Santanghu Basin & Lucaogou & Lacustrine & Dolomite \\
\hline & Yangzte area & & Marine & $\begin{array}{c}\text { Limestone } \\
\text { Silicolite }\end{array}$ \\
\hline & Southwest China & & Marine & Silicolite \\
\hline & Sichuan Basin & Dalong & Marine & Silicolite \\
\hline & Guangxi area & Maokou & Marine & Silicolite \\
\hline & Middle-lower Yangtze & & Marine & Silicolite \\
\hline Devomian & Yunnan area & Changyucun & Marine & Silicolite \\
\hline \multirow{2}{*}{ Silurian } & Middle-upper Yangtze & Longmaxi & Marine & Carbonaceous shale \\
\hline & Southeast Sichuan & Longmaxi & Marine & Carbonaceous shale \\
\hline \multirow{12}{*}{ 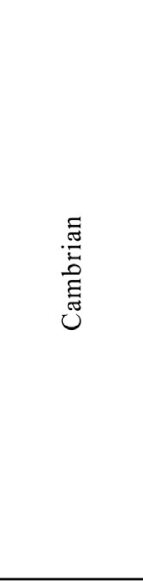 } & Tarim Basin & Yuertusi & Marine & Phosphoric silicolite \\
\hline & Upper Yangtze & Niutitang & Marine & Dolomite \\
\hline & Beiyuan area & & Marine & Limistone \\
\hline & West Hunan & Niutitang & Marine & Carbonaceous shale \\
\hline & Tongren area & Niutitang & Marine & $\begin{array}{c}\text { Silicolite } \\
\text { Black shale }\end{array}$ \\
\hline & Hunan area & Niutitang & Marine & Silicolite \\
\hline & Northwest Guizhou & Niutitang & Marine & Black shale \\
\hline & North Guizhou & Niutitang & Marine & Black shale \\
\hline & East Guizhou & Niutitang & Marine & Black shale \\
\hline & Changyang area & Niutitang & Marine & Black shale \\
\hline & Southeast Sichuan & Niutitang & Marine & Siliceous shale \\
\hline & Shitai area & Hetang & Marine & Black shale \\
\hline \multirow{5}{*}{ 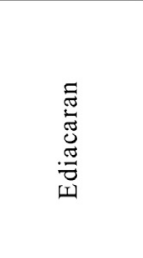 } & West Hubei & Doushantuo & Marine & Dolomite \\
\hline & East Chongqing & Dengying & Marine & Silicolite \\
\hline & Southeast Chongqing & Dengying & Marine & $\begin{array}{l}\text { Silicolite } \\
\text { Shale }\end{array}$ \\
\hline & Xiahuayuan area & Xiamaling & Marine & Black shale \\
\hline & Mentougou area & Xiamaling & Marine & Black shale \\
\hline
\end{tabular}

FIGURE 1 | Stratigraphic distribution of source rocks related to hydrothermal activity in China (modified after Jia et al., 2016).

and Cronan, 1995; Zheng et al., 2006; Dekov et al., 2011; Wen et al., 2013; Slack et al., 2015; He et al., 2016; He et al., 2017; Liu et al., 2017; Liu et al., 2021a; Liu et al., 2021b). Hydrothermal sediments are often the focus of geological research due to their enrichment in metallic elements (e.g., Fe, Mn, Cu, $\mathrm{Zn}, \mathrm{Au}, \mathrm{Ag}, \mathrm{Sn}$, etc.) (Dias and Barriga, 2006; Wen et al., 2013; Li et al., 2014; Zhong et al., 2015; He et al., 2016). Many indicators for hydrothermal activity have been proposed and established including a combination of special minerals and rocks, sedimentary structures, major and trace element 
discrimination diagrams, rare earth element (REE) content and distribution, and stable isotope composition (He et al., 2016; Jia et al., 2016).

Oil-gas exploration has shown that the formation of many black shales (or organic-rich source rocks) is closely related to hydrothermal activities, such as the Lower Cambrian marine source rocks in Tarim Basin, the Neoproterozoic Xiamaling Formation black shale in North China, and the Lower Cambrian Niutitang Formation marine organic-rich shales in Yangtze Platform (Figure 1). This relationship is demonstrated in two ways: 1) hydrothermal activity areas are often accompanied with the occurrence of contemporaneous organic-rich source rocks; and 2) abnormal metal elements caused by hydrothermal activities display linear relationship with the total organic carbon (TOC) contents of organic-rich source rocks (Zhang et al., 2010; Jia et al., 2016). Zhang et al. (2010) suggested that the submarine or sublacustrine hydrothermal activities would significantly change sedimentary environments (e.g., water temperature, redox conditions, and chemical composition of water column), further affecting surface primary productivity and preservation of organic matter. Previous studies on modern hydrothermal systems have observed diverse biological communities near hydrothermal vents, such as hydrothermal areas in the Galapagos, where its biological yield is 3.9 times of the ordinary ocean surface, and the Fiji Basin, where both quantity and metabolic activity are 1-3 orders of magnitude higher than the ocean's surface (Karl et al., 1980; Van Dever et al., 1996; Pichler and Dix, 1996; Halbach et al., 2001; Tarasov et al., 2005 and references herein; Wang et al., 2006; Hey et al., 2006; Wang et al., 2008; Liang et al., 2014a; Liang et al., 2014b). This level of biological activity suggests that hydrothermal activity may play an important role in promoting marine or lacustrine primary productivity. Prior research has examined the relationship between hydrothermal activity and the formation of organic-rich shales, and has obtained valuable information (Sun et al., 2003; Chen et al., 2004; Zhang et al., 2010; Liang et al., 2014a; Liang et al., 2014b; He et al., 2017). However, our understanding of this relationship is still relatively lacking and the topic itself has not attracted enough attention (Jia et al., 2016). Therefore, it is necessary to continue exploring the potential influence of hydrothermal activity on organic matter accumulation in order to add to existing knowledge of formation mechanisms of organic-rich shale, and oil-gas exploration and development.

The Chang 7 lacustrine black shale in the Upper Triassic Yanchang Formation is an important hydrocarbon source rock and contains high TOC concentrations, providing the main source of oil for the Mesozoic petroleum system in the Ordos Basin (Yang and Zhang, 2005; Yuan et al., 2017). Additionally, several contemporaneous special minerals and rocks, including tuff interval, seismite, seismoturbidite, silicolite, and microcrystalline dolomite lamina, are found within the Chang 7 black shale and associated with significant geological events (Zhang et al., 2017). Therefore, it has been proposed that the accumulation of organic matter during the deposition of the Chang 7 shale is related to these geological events (Zhang et al., 2009; Yang et al., 2010; Zhang et al., 2010; Qiu, 2011; Qiu et al.,
2014; Qiu et al., 2015; Zhang et al., 2017). In recent years, there have been significant mineralogical, petrological, elemental, and isotopic evidences of hydrothermal sedimentation in the Chang 7 Member of Yanchang Formation. This suggests that hydrothermal activity may play an important role in the accumulation of organic matter (Zhang et al., 2010; Qiu et al., 2015; He et al., 2016; He et al., 2017). However, we still have a limited understanding of the relationship between hydrothermal activity and the accumulation of organic matter due to the lack of abundant and available data. In this paper, we present mineralogical evidence for hydrothermal activity (as supplementary for previous reports) and a collection of geochemical data (including TOC, major and trace element contents) that together, explores the potential relationship between hydrothermal activity and the accumulation of organic matter during the deposition of the Chang 7 black shale.

\section{GEOLOGICAL SETTING}

The Ordos Basin, located in central China (Figure 2A), is a typical multicycle cratonic depression basin developed on Archean granulites and Lower Proterozoic greenschists, consisting of six tectonic units (Figure 2B) (Yuan et al., 2017; He et al., 2017). In the Early-Middle Triassic, the Ordos Basin was part of the North China intracratonic depression (Qiu et al., 2015). By the Late Triassic, the North China landmass collided and integrated with the Yangtze landmass, leading to the closure of the relict of the Youjiang and Qinling Troughs and the uplift of the Qinling Mountains (also called Qinling orogenic movement) (Zhang et al., 2010; Zhang et al., 2017). This was responsible for the formation of many basement faults in the periphery and interior of the Ordos Basin (Figure 2B). The Qinling orogenic movement transformed Ordos Basin into a foreland basin, which was characterized in its asymmetric cross-section by low-gradient northeastern and highgradient southwestern flanks (Qiu et al., 2015). This tectonic process provided a large depositional space and subsequently deposited a set of clastic sediments in the Late Triassic, known as the Yanchang Formation, with a thickness of about 1,000-1,300 m (Wang et al., 2017). This unit can be divided into 10 members (Chang 1-Chang 10) from top to bottom (Qiu et al., 2014; Qiu et al., 2015; He et al., 2016; Wang et al., 2017; Yuan et al., 2017; Zhang et al., 2017), and the Chang seven Member can be further subdivided into 3 submembers, Chang $7_{1}$ to Chang $7_{3}$, from top to bottom (Wang et al., 2017). In the early stages of the deposition of Chang 7 Member, the lacustrine basin subsided and expanded rapidly due to the intensely tectonic movement coupled with frequent volcanic and seismic activities, which provided the necessary conditions for reactivation of basement faults and the upwelling of deep hydrothermal fluids (Deng et al., 2008; He et al., 2016; Zhang et al., 2017). During this time period, the Chang 7 black shale was deposited and became the predominant oil-source rocks for the Mesozoic of this basin (Yang and Zhang, 2005; Zhang et al., 2010). 


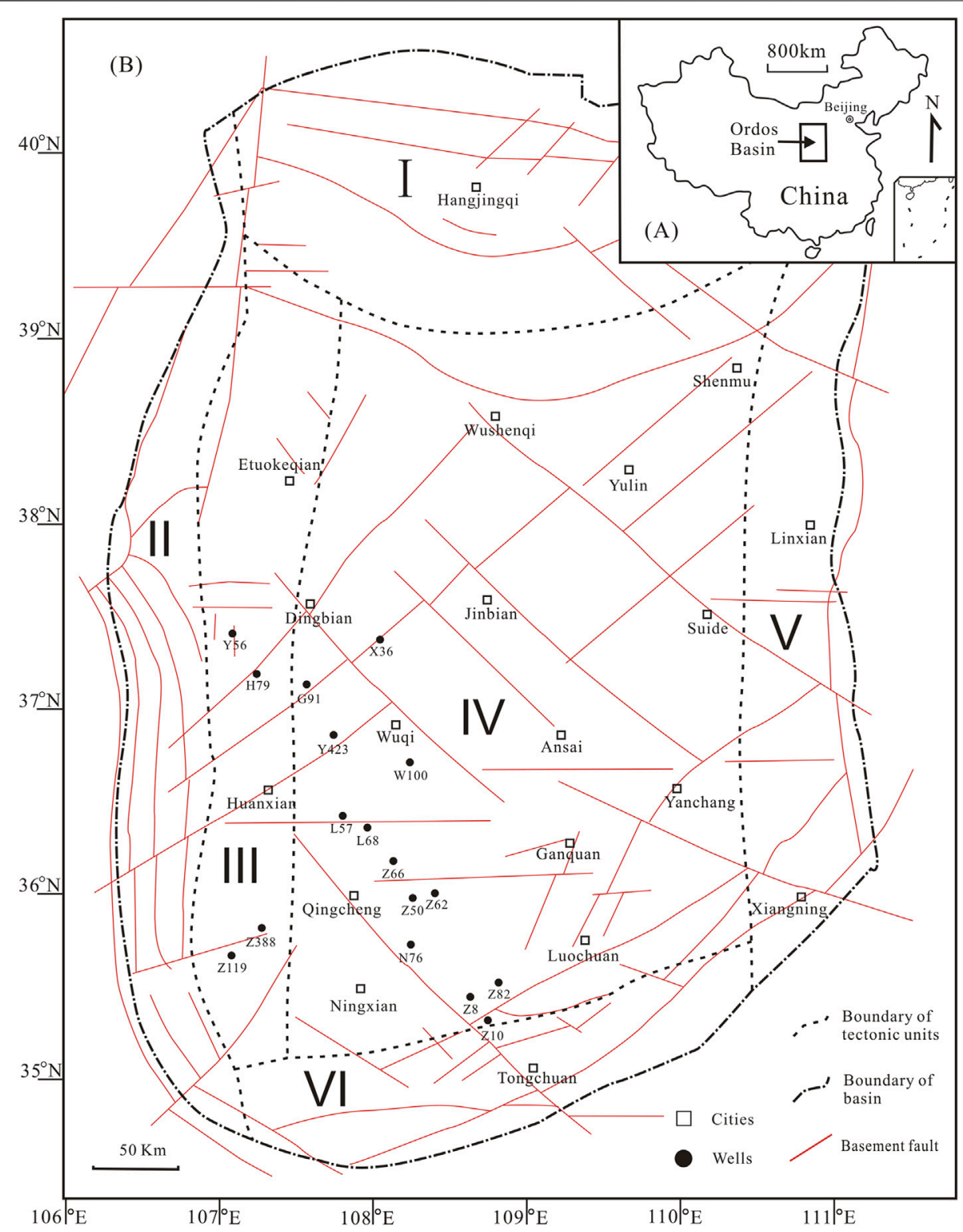

FIGURE 2 | Location of the Ordos Basin, its tectonic division, distribution of basement faults, and the location of sample wells (modified after Qiu, 2011). I-Yimeng uplift; II-West margin thrust belts; III-Tianhuan depression; IV-Yishan slope; V-Jinxi flexure belts; VI-Weibei uplift.

\section{SAMPLES AND ANALYTICAL METHODS}

Many Chang 7 drill cores were observed and pictures were taken at the Xifeng drill core stores of the PetroChina Changqing Oilfield Company, southeastern Gansu Province, northwest China. A total of 78 Chang 7 black shale samples were collected from 17 wells, which are located in the southwestern Ordos Basin (Figure 2B). To minimize the effect of volcanic ashes, tuffs were carefully avoided when selecting the samples.

Rock thin-sections of the Chang 7 shale samples were made at the State Key Laboratory of Petroleum Resource and Prospecting, China University of Petroleum-Beijing (CUPB), and were observed under an optical microscope (LEICA DM4500P) in order to identify their mineral compositions. A subsample of shale sample fragments with freshly broken surfaces were selected and coated with gold, and then observed using a Quanta 200F field emission scanning electron microscope (SEM) equipped with an energy-dispersive X-ray spectrometer (EDS) at the Microstructure Laboratory for Energy Materials of CUPB.

All of the black shale samples were crushed to powder (less than $200 \mathrm{mesh}$ ) in an agate mortar prior to geochemical analyses. TOC contents were measured at the State Key Laboratory of Petroleum Resource and Prospecting of CUPB. Approximately $100 \mathrm{mg}$ of shale powder was gently leached with dilute hydrochloric acid $(\mathrm{HCl})$ and rinsed with distilled water to remove inorganic carbon. After drying, the shale powders 

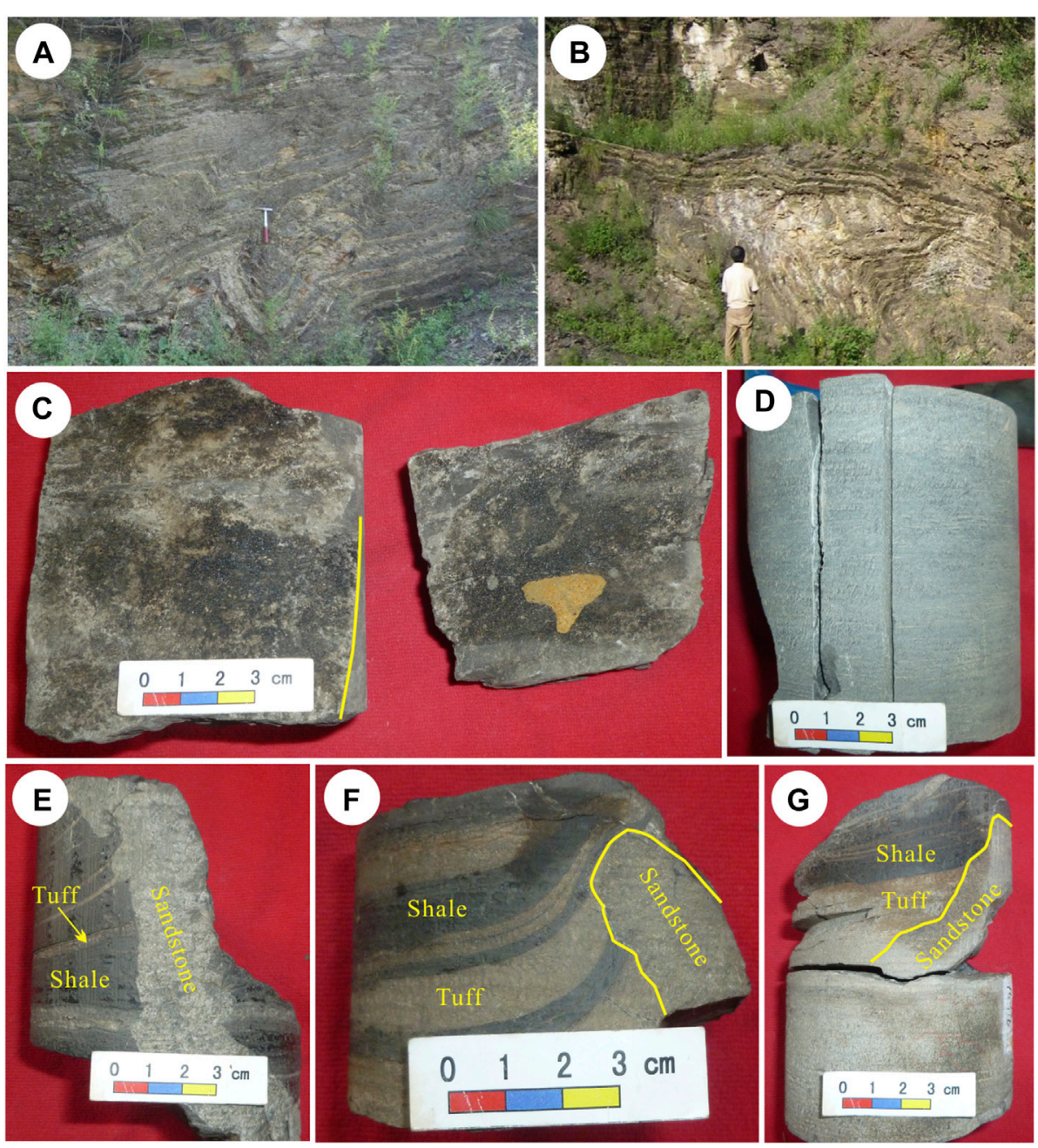

FIGURE 3 | Outcrop and core photographs of the Chang 7 Member in the Ordos Basin $(\mathbf{A}, \mathbf{B})$ Folds in the Chang 7 black shale in the west of Hejiafang section, Tongchuan (after Qiu, 2011); (C) High angle fracture with residual asphalt, Well Z119, $2241.30 \mathrm{~m}$; (D) Vertical fractures, Well Z140, 1884.70 m; (E-G) Tuff intervals, and structural deformations caused by earthquake, Well W100, the depth is $1914.45 \mathrm{~m}, 1914.73$ and $1914.95 \mathrm{~m}$, respectively.

were analyzed using an organic carbon analyzer (LECO CS-230) and the analytical uncertainly is $<3 \%$.

Major and trace element concentrations of 68 shale samples were measured at the Analytical Laboratory of Beijing Research Institute of Uranium Geology. Major element oxides were determined by a PHILIPS PW2404 X-ray fluorescence spectrometer (XRF), following analytical methods reported by Ma et al. (2015). Trace elements compositions were measured using a Thermo Scientific Element XR inductively coupled plasma-mass spectrometry (ICP-MS) according to Gao et al. (2015). The analytical precision is $<5 \%$ for all major elements, while the precision and accuracy are estimated to be $<10 \%$ for the trace elements.

The $\delta^{13} \mathrm{C}$ and $\delta^{18} \mathrm{O}$ analyses of bulk carbonates for ten black samples and one carbonate vein were carried out at the State Key Laboratory of Petroleum Resource and Prospecting of CUPB. Approximately $10 \mathrm{mg}$ sample powders were reacted with phosphoric acid $\left(\mathrm{H}_{3} \mathrm{PO}_{4}\right)$ and liberated $\mathrm{CO}_{2}$ were analyzed for $\delta^{13} \mathrm{C}$ and $\delta^{18} \mathrm{O}$ using a Therma Fisher MAT 253 isotope ratio mass spectrometer (IR-MS). The results are presented using delta notation in reference to the PDB standard. Repeated measurements of a homogenized sample yielded a standard deviation of $\pm 0.1 \%$ or for $\delta^{13} \mathrm{C}$ and $\delta^{18} \mathrm{O}$ measurements.

\section{RESULTS}

\section{Mineral Petrography}

Outcrop and core observations reveal the occurrence of folds (Figures 3A,B), high angle fractures (Figures 3C,D), structural deformations, and tuff intervals (Figures 3E-G) in the Chang 7 Member of Yanchang Formation, indicating frequent tectonic and volcanic activity. In addition, pyrite veins (Figures 4A-D), carbonate veins, and carbonate laminae (Figures $\mathbf{4 E}-\mathbf{H}$ ) were 

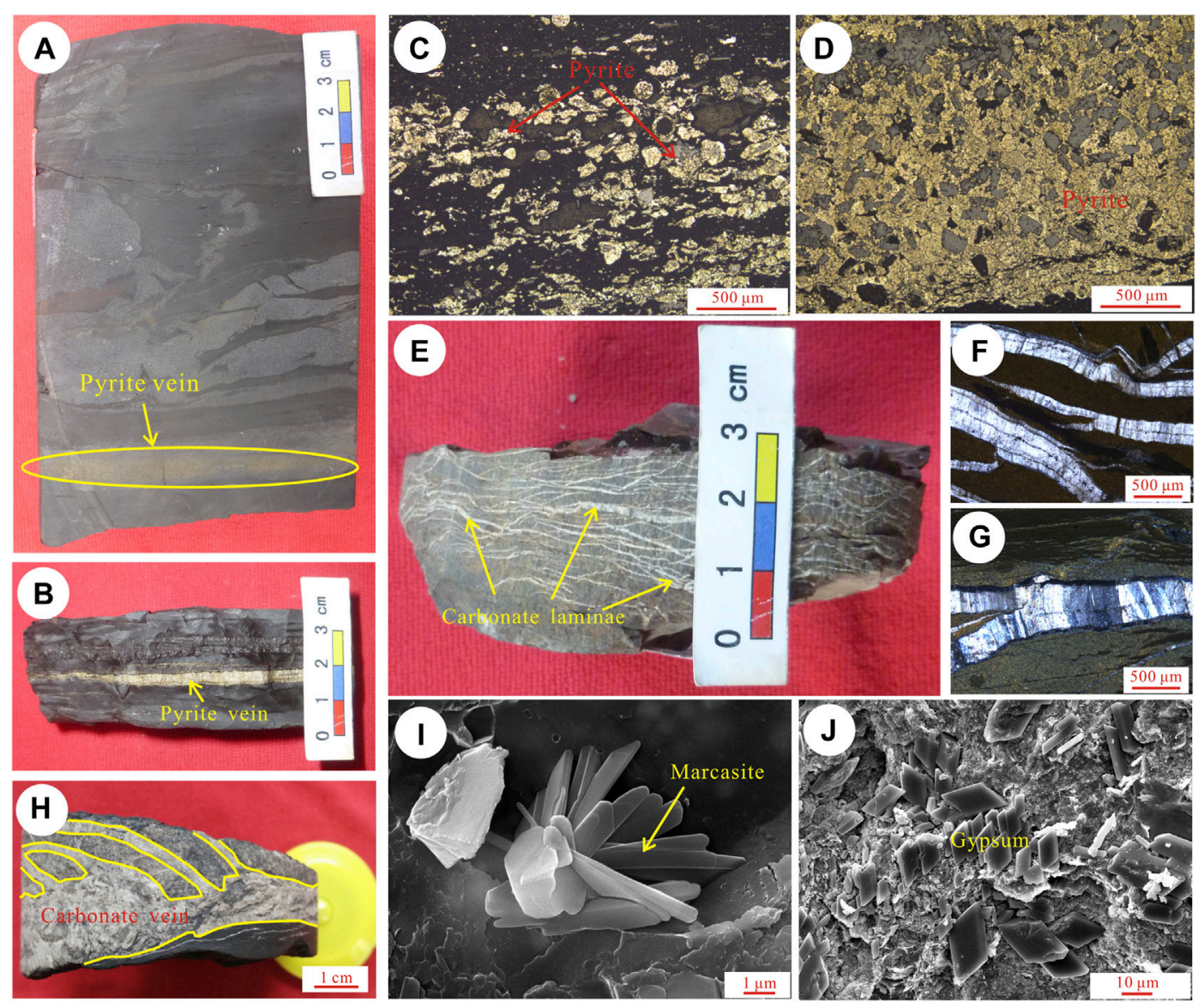

FIGURE 4 | Photographs of minerals in the Chang 7 black shale of the Ordos Basin (A) Pyrite vein, Well L57, 2350.13 m; (B) Pyrite vein, Well X36, 2188.58 m; (C,D) Microscopic characteristics of pyrite vein in photo B; (E) Carbonate laminae, Well W100, 1907.05 m; (F,G) Microscopic characteristics of carbonate laminae in photo E; (H) Carbonate vein characterized by branches, Well Y56, 3065.24 m; (I) Marcasite, Well Z62, 1938.00 m; (J) Gypsum, Well Y423, $2402.17 \mathrm{~m}$.

found in this unit. Microscopic observations of pyrite veins showed heterogeneous and amorphous characteristics (Figures 4C,D), with many impurities (the grey part in Figure 4D). The carbonate veins were characterized by branches (Figure $\mathbf{4 H}$ ), and a lot of bubbles formed after dilute $\mathrm{HCl}$ was added. Some special minerals, such as marcasite (Figure 4I) and gypsum (Figure 4J), were also observed through SEM observations.

\section{TOC, $\delta^{13} \mathrm{C}$ and $\delta^{18} \mathrm{O}$}

TOC contents of the 68 Chang 7 black shale samples were very variable, ranging from 0.63 to $29.93 \%$ and an average of $13.47 \%$ (Table 1). TOC contents were mainly distributed between 2 and 20\%, accounting for $75 \%$ of the total (Figure 5), indicating a high abundance of organic matter and good to excellent hydrocarbon generation potential.

The $\delta^{13} \mathrm{C}$ and $\delta^{18} \mathrm{O}$ data of carbonates in 11 samples are presented in Table 2. The $\delta^{13} \mathrm{C}$ and $\delta^{18} \mathrm{O}$ values of bulk carbonates for 10 black samples varied from -1.4 to
$1.6 \%$ (mean $=0.8 \%$ ) and from $-11.1 \%$ to $-7.5 \%$ ( $-9.4 \%$ ), respectively. However, the carbonate vein in Figure 4E showed lower $\delta^{13} \mathrm{C}(-8.2 \%)$ and $\delta^{18} \mathrm{O}(-20.0 \%)$ values than that of bulk carbonates of the Chang 7 black shale.

\section{Major and Trace Elements}

Selected major oxides (including $\mathrm{TiO}_{2}, \mathrm{SiO}_{2}, \mathrm{Al}_{2} \mathrm{O}_{3}, \mathrm{Fe}_{2} \mathrm{O}_{3}$, $\mathrm{MnO}$, and $\mathrm{P}_{2} \mathrm{O}_{5}$ ) are listed in Table $1 . \mathrm{SiO}_{2}, \mathrm{Al}_{2} \mathrm{O}_{3}$ and $\mathrm{Fe}_{2} \mathrm{O}_{3}$ were the dominant components of shale samples, with average contents of $48.95 \%, 13.35 \%$, and $10.09 \%$, respectively. Contents of $\mathrm{TiO}_{2}, \mathrm{MnO}$, and $\mathrm{P}_{2} \mathrm{O}_{5}$ were less than $1 \%$, except for a few samples, with average contents of $0.49 \%$, $0.09 \%$ and $0.49 \%$, respectively. Contents of $\mathrm{Ti}, \mathrm{Al}, \mathrm{Fe}, \mathrm{Mn}$, and $\mathrm{P}$ were calculated based on their molecular formula, with mean values of $0.29 \%, 7.07 \%, 7.06 \%, 0.07 \%$ and $0.21 \%$, respectively (Table 1). Concentrations of trace elements are also presented in Table 1. Average trace element contents of the Chang 7 black shale were normalized to the post-Archean Australian 
TABLE 1 | Selected major, trace elements data and geochemical indicators of the Chang 7 black shale in Ordos Basin.

\begin{tabular}{|c|c|c|c|c|c|c|c|c|c|c|c|c|c|c|c|c|c|c|c|c|c|c|}
\hline \multirow[t]{2}{*}{ No } & \multirow[t]{2}{*}{ Wells } & \multirow{2}{*}{$\begin{array}{l}\text { Depth } \\
\text { (m) }\end{array}$} & TOC & $\mathrm{TiO}_{2}$ & $\mathrm{SiO}_{2}$ & $\mathrm{Al}_{2} \mathrm{O}_{3}$ & $\mathrm{Fe}_{2} \mathrm{O}_{3}$ & MnO & $\mathrm{P}_{2} \mathrm{O}_{5}$ & $\mathrm{Ti}$ & Al & $\mathrm{Fe}$ & Mn & $\mathbf{P}$ & $\mathrm{Ba}$ & $\mathrm{Sr}$ & $\mathrm{Cr}$ & Co & $\mathrm{Ni}$ & $\mathrm{Cu}$ & $\mathrm{Zn}$ & Ga \\
\hline & & & \multicolumn{12}{|c|}{ (\%) } & \multicolumn{8}{|c|}{ (ppm) } \\
\hline 1 & L57 & 2330.10 & 10.50 & 0.57 & 55.91 & 14.81 & 5.91 & 0.12 & 0.26 & 0.34 & 7.84 & 4.13 & 0.09 & 0.11 & 442.7 & 80.9 & 88.7 & 20.4 & 42.0 & 109.8 & 83.1 & 19.5 \\
\hline 2 & L57 & 2331.60 & 12.76 & 0.65 & 57.87 & 16.39 & 6.53 & 0.04 & 0.31 & 0.39 & 8.68 & 4.57 & 0.03 & 0.14 & 542.5 & 107.7 & 92.6 & 19.8 & 42.4 & 91.8 & 93.6 & 20.7 \\
\hline 3 & L57 & 2333.55 & 12.09 & 0.56 & 57.72 & 16.14 & 7.20 & 0.04 & 0.38 & 0.34 & 8.54 & 5.03 & 0.03 & 0.17 & 549.0 & 92.9 & 89.5 & 22.4 & 45.0 & 113.0 & 92.3 & 21.6 \\
\hline 4 & L57 & 2335.48 & 14.80 & 0.64 & 57.46 & 15.84 & 6.36 & 0.05 & 0.33 & 0.39 & 8.39 & 4.45 & 0.04 & 0.15 & 588.1 & 135.4 & 92.9 & 19.9 & 43.2 & 108.0 & 91.3 & 20.4 \\
\hline 5 & L57 & 2337.15 & 17.25 & 0.47 & 54.21 & 13.54 & 10.20 & 0.02 & 0.37 & 0.28 & 7.17 & 7.13 & 0.01 & 0.16 & 589.6 & 162.8 & 67.5 & 21.4 & 45.9 & 127.4 & 89.5 & 17.9 \\
\hline 6 & L57 & 2338.80 & 9.21 & 0.49 & 55.79 & 14.37 & 8.94 & 0.02 & 0.35 & 0.30 & 7.61 & 6.25 & 0.01 & 0.15 & 604.1 & 183.5 & 69.6 & 20.1 & 43.4 & 120.7 & 93.8 & 18.6 \\
\hline 7 & L57 & 2340.94 & 16.30 & 0.57 & 54.84 & 15.82 & 6.82 & 0.02 & 0.36 & 0.34 & 8.38 & 4.77 & 0.02 & 0.16 & 639.4 & 158.1 & 96.5 & 22.7 & 44.2 & 155.7 & 100.6 & 21.1 \\
\hline 8 & L57 & 2342.35 & 14.30 & 0.65 & 60.07 & 14.52 & 5.36 & 0.02 & 0.55 & 0.39 & 7.69 & 3.75 & 0.02 & 0.24 & 697.2 & 225.8 & 77.4 & 21.9 & 38.3 & 121.5 & 90.7 & 19.2 \\
\hline 9 & L57 & 2344.50 & 14.09 & 0.56 & 61.15 & 14.91 & 6.06 & 0.02 & 0.36 & 0.34 & 7.89 & 4.24 & 0.02 & 0.16 & 769.4 & 212.1 & 86.6 & 24.0 & 38.7 & 136.4 & 93.7 & 20.7 \\
\hline 10 & L57 & 2346.70 & 19.55 & 0.35 & 43.23 & 11.93 & 15.54 & 0.06 & 0.61 & 0.21 & 6.32 & 10.87 & 0.04 & 0.27 & 475.1 & 204.0 & 66.3 & 22.0 & 54.0 & 232.8 & 110.5 & 17.0 \\
\hline 11 & L57 & 2348.20 & 20.68 & 0.40 & 49.55 & 13.75 & 11.23 & 0.35 & 1.01 & 0.24 & 7.28 & 7.85 & 0.27 & 0.44 & 714.4 & 333.7 & 80.9 & 25.3 & 58.5 & 294.1 & 126.1 & 19.7 \\
\hline 12 & L57 & 2349.50 & 26.18 & 0.46 & 57.06 & 14.26 & 7.54 & 0.02 & 0.51 & 0.27 & 7.55 & 5.27 & 0.02 & 0.22 & 816.0 & 276.9 & 64.2 & 23.3 & 40.9 & 131.8 & 96.3 & 18.8 \\
\hline 13 & L57 & 2352.95 & 11.01 & 0.62 & 56.16 & 16.74 & 6.16 & 0.03 & 0.40 & 0.37 & 8.86 & 4.31 & 0.02 & 0.18 & 876.0 & 286.5 & 75.8 & 23.4 & 40.6 & 106.4 & 107.6 & 20.4 \\
\hline 14 & L57 & 2353.79 & 15.30 & 0.48 & 54.90 & 14.83 & 7.47 & 0.03 & 0.90 & 0.29 & 7.85 & 5.22 & 0.02 & 0.39 & 788.1 & 390.8 & 55.0 & 23.5 & 39.8 & 120.2 & 99.6 & 17.4 \\
\hline 15 & L57 & 2318.45 & 3.35 & 0.65 & 55.12 & 14.27 & 6.02 & 0.06 & 0.20 & 0.39 & 7.55 & 4.21 & 0.05 & 0.09 & & 93.9 & 101.0 & 14.5 & 27.3 & 57.2 & 79.2 & 18.1 \\
\hline 16 & L68 & 1998.40 & 7.80 & 0.65 & 48.95 & 17.26 & 8.46 & 0.17 & 0.2 & 0.3 & 9.14 & 5.9 & 0.13 & 0.1 & 721.3 & 110.5 & 79.3 & 22.0 & 42.2 & 108.8 & & 23.0 \\
\hline 17 & L68 & 2071.40 & 9.61 & 0.55 & 42.99 & 14.80 & 13.00 & 0.05 & 0.60 & 0.33 & 7.84 & 9.09 & 0.04 & 0.26 & 399.2 & 143.0 & 63.0 & 34.4 & 51.1 & 188.7 & 118.2 & 21.6 \\
\hline 18 & L68 & 2076.60 & 15.61 & 0.55 & 40.27 & 13.41 & 10.38 & 0.08 & 0.46 & 0.33 & 7.10 & 7.26 & 0.06 & 0.20 & 464.0 & 108.6 & 62.0 & 25.0 & 36.8 & 112.1 & 93.3 & 18.0 \\
\hline 19 & L68 & 2078.20 & 16.79 & 0.60 & 47.28 & 11.26 & 7.12 & 0.06 & 0.45 & 0.36 & 5.96 & 4.98 & 0.05 & 0.20 & 374.3 & 116.8 & 56.0 & 21.7 & 29.2 & 120.2 & 82.3 & 17.6 \\
\hline 20 & L68 & 2080.10 & 28.39 & 0.20 & 35.25 & 7.23 & 14.85 & 0.35 & 0.69 & 0.12 & 3.83 & 10.38 & 0.27 & 0.3 & 321.6 & 154.6 & 26.1 & 22.5 & 41.3 & 242.6 & 87.4 & 14.1 \\
\hline 21 & L68 & 2082.30 & 26.82 & 0.25 & 45.23 & 6.05 & 8.46 & 0.33 & 0.7 & 0.15 & 3.20 & 5.92 & 0.26 & 0. & 477.1 & 185.3 & 17.6 & 28.1 & 33.9 & 207.7 & 81.3 & 12.1 \\
\hline 22 & N76 & 1729.10 & 1.86 & 0.38 & 69.49 & 13.54 & 5.77 & 0.01 & 0.15 & 0.23 & 7.17 & 4.03 & 0.01 & 0.06 & 687.0 & 140.0 & 57.8 & 11.4 & 18.9 & 42.2 & 76.5 & 20.9 \\
\hline 23 & N76 & 1727.35 & 0.63 & 0.77 & 57.13 & 17.52 & 9.57 & 0.06 & 0.17 & 0.46 & 9.28 & 6.69 & 0.05 & 0.08 & 746.0 & 149.0 & 83.6 & 16.5 & 32.6 & 44.6 & 106.0 & 22.6 \\
\hline 24 & N76 & 1716.60 & 0.77 & 1.00 & 49.98 & 20.93 & 9.95 & 0.05 & 0.17 & 0.60 & 11.08 & 6.96 & 0.04 & 0.07 & 881.0 & 170.0 & 115.0 & 23.5 & 42.1 & 53.8 & 107.0 & 28.7 \\
\hline 25 & Z8 & 1262.2 & 2.35 & 0.73 & 56.59 & 15.95 & 5.91 & 0.07 & 0.16 & 0.44 & 8.44 & 4.13 & 0.06 & 0.07 & 609.0 & 142.0 & 75.7 & 15.5 & 45.1 & 39.0 & 111.0 & 20.1 \\
\hline 26 & $Z \varepsilon$ & 1263.0 & 19.18 & 0.30 & 23.89 & 8.48 & 17.25 & 0.04 & 0.27 & 0.18 & 4.49 & 12.06 & 0.03 & 0.1 & 347.0 & 69.7 & 43.7 & 27.8 & 57.2 & 206.0 & 116.0 & 13.2 \\
\hline 27 & Z8 & 1264.50 & 3.25 & 0.70 & 50.83 & 17.42 & 8.62 & 0.05 & 0.16 & 0.42 & 9.22 & 6.03 & 0.04 & 0.07 & 463.0 & 117.0 & 73.9 & 23.6 & 45.9 & 76.2 & 119.0 & 21.7 \\
\hline 28 & Z8 & 1267.9-1 & 5.92 & 0.35 & 43.60 & 13.96 & 10.22 & 0.04 & 0.22 & 0.21 & 7.39 & 7.15 & 0.03 & 0.10 & 400.0 & 88.4 & 59.6 & 21.4 & 43.0 & 117.0 & 121.0 & 17.5 \\
\hline 29 & Z8 & $1267.9-2$ & 24.29 & 0.57 & 29.81 & 11.13 & 13.26 & 0.02 & 0.18 & 0.34 & 5.89 & 9.27 & 0.02 & $0 . c$ & 259.0 & 73.1 & 50.0 & 25.0 & 43.2 & 157.0 & 109.0 & 14.6 \\
\hline 30 & Z8 & 1276.45 & 24.01 & 0.40 & 25.99 & 7.51 & 16.45 & 0.04 & 0.22 & 0.24 & 3.98 & 11.50 & 0.03 & 0.09 & 246.0 & 86.1 & 50.2 & 24.4 & 44.8 & 209.0 & 103.0 & 11.6 \\
\hline 31 & Z8 & 277.20 & 19.07 & 0.4 & 29.7 & 7.2 & 15.52 & 0.05 & 0.22 & 0.27 & 3.83 & 10.85 & 0.04 & 0.0 & 300.0 & 89.9 & 29.3 & 20.5 & 35.8 & 166.0 & 97.1 & 10.6 \\
\hline 32 & Z8 & 1278.50 & 23.15 & 0.30 & 31.23 & 9.22 & 14.88 & 0.05 & 0.29 & 0.18 & 4.88 & 10.40 & 0.04 & 0.13 & 243.0 & 131.0 & 38.4 & 21.7 & 40.9 & 179.0 & 93.9 & 12.9 \\
\hline 33 & Z8 & 1278.80 & 29.93 & 0.30 & 28.09 & 7.17 & 17.25 & 0.09 & 0.30 & 0.18 & 3.80 & 12.06 & 0.07 & 0.13 & 222.0 & 100.0 & 28.0 & 16.3 & 33.7 & 165.0 & 91.0 & 10.6 \\
\hline 34 & Z8 & 1279.50 & 9.43 & 0.57 & 47.12 & 15.63 & 9.74 & 0.04 & 0.18 & 0.34 & 8.27 & 6.81 & 0.03 & 0.08 & 694.0 & 134.0 & 39.0 & 19.0 & 26.4 & 77.7 & 96.9 & 18.0 \\
\hline 35 & Z8 & 80.40 & 25.50 & 0.45 & 28.30 & 8.80 & 13.77 & 0.21 & 0.56 & 0.27 & 4.66 & 9.63 & 0.16 & 0.2 & 339.0 & 151.0 & 44.5 & 19.2 & 39.0 & 180.0 & 110.0 & 13.9 \\
\hline 36 & Z8 & 81.70 & 28.27 & 0.45 & 31.23 & 10.26 & 14.02 & 0.04 & 0.44 & 0.27 & 5.43 & 9.80 & 0.03 & 0.1 & 438.0 & 189.0 & 49.3 & 22.2 & 41.9 & 166.0 & 104.0 & 15.2 \\
\hline 37 & Z8 & 1283.00 & 18.96 & 0.48 & 50.97 & 14.63 & 7.86 & 0.16 & 0.18 & 0.29 & 7.75 & 5.50 & 0.12 & 0.08 & 621.0 & 249.0 & 45.4 & 18.7 & 26.1 & 84.1 & 94.7 & 18.2 \\
\hline 38 & Z8 & 1284.05 & 21.61 & 0.45 & 43.47 & 11.78 & 11.24 & 0.03 & 0.27 & 0.27 & 6.24 & 7.86 & 0.03 & 0.12 & 450.0 & 141.0 & 61.2 & 19.1 & 32.0 & 124.0 & 94.9 & 16.0 \\
\hline 39 & Z8 & 1284.70 & 8.65 & 0.50 & 40.75 & 14.94 & 11.31 & 0.61 & 0.22 & 0.30 & 7.91 & 7.91 & 0.47 & 0.10 & 563.0 & 208.0 & 57.0 & 20.2 & 34.3 & 118.0 & 106.0 & 21.7 \\
\hline 40 & Z8 & 1287.70 & 12.33 & 0.50 & 40.39 & 11.80 & 13.10 & 0.46 & 0.44 & 0.30 & 6.25 & 9.16 & 0.35 & 0.1 & 895.0 & 392.0 & 53.2 & 20.7 & 40.2 & 142.0 & 93.7 & 17.1 \\
\hline 41 & $Z 50$ & 1320 & 27.50 & 0.31 & 45.66 & 9.1 & 14.58 & 0.24 & 0.71 & 0.19 & 4.84 & 10.20 & 0.19 & 0.3 & 333.0 & 194.3 & & 28.2 & 47.3 & 373 & 113.1 & 13.8 \\
\hline 42 & Z50 & 1943.80 & 8.30 & 0.28 & 76.27 & 9.33 & 3.95 & 0.18 & 0.24 & 0.17 & 4.94 & 2.76 & 0.14 & 0.10 & 742.3 & 212.3 & 29.7 & 11.1 & 21.0 & 398.1 & 66.7 & 10.0 \\
\hline 43 & $Z 50$ & 1944.07 & 24.97 & 0.40 & 44.15 & 10.28 & 13.20 & 0.20 & 0.61 & 0.24 & 5.44 & 9.23 & 0.16 & 0.26 & 664.9 & 227.1 & 18.3 & 24.8 & 43.7 & 295.7 & 112.4 & 16.2 \\
\hline 44 & $Z 50$ & 1945.60 & 18.34 & 0.40 & 51.11 & 12.03 & 13.90 & 0.09 & 0.83 & 0.24 & 6.37 & 9.72 & 0.07 & 0.36 & 659.8 & 243.9 & 29.4 & 22.9 & 37.6 & 382.0 & 105.3 & 15.0 \\
\hline 45 & Z50 & 1946.00 & 8.41 & 0.49 & 70.89 & 14.58 & 2.97 & 0.08 & 0.09 & 0.30 & 7.72 & 2.08 & 0.06 & 0.0 & 687.3 & 268.9 & 55.0 & 6.0 & 13.6 & 457.9 & 84.2 & 16.9 \\
\hline 46 & Z62 & & 19.40 & & 35.66 & 9.64 & 19.15 & 0.16 & 0.54 & & 5.10 & 13.39 & 0.12 & & 556.0 & 264.0 & 40.2 & 22.0 & 43.0 & 200.0 & 109.0 & 15.9 \\
\hline 47 & Z62 & 1938.00 & 13.00 & 0.42 & 41.16 & 12.97 & 15.99 & 0.05 & 0.67 & 0.25 & 6.87 & 11.18 & 0.04 & 0.29 & 570.0 & 395.0 & 65.2 & 25.6 & 53.2 & 227.0 & 118.0 & 19.1 \\
\hline 48 & Z66 & 2047.04 & 12.26 & 0.21 & 45.54 & 7.68 & 16.37 & 0.03 & 0.75 & 0.13 & 4.07 & 11.45 & 0.02 & 0.33 & 139.4 & 107.9 & 35.4 & 18.1 & 43.1 & 191.2 & 100.8 & 13.4 \\
\hline
\end{tabular}


TABLE 1 | (Continued) Selected major, trace elements data and geochemical indicators of the Chang 7 black shale in Ordos Basin.

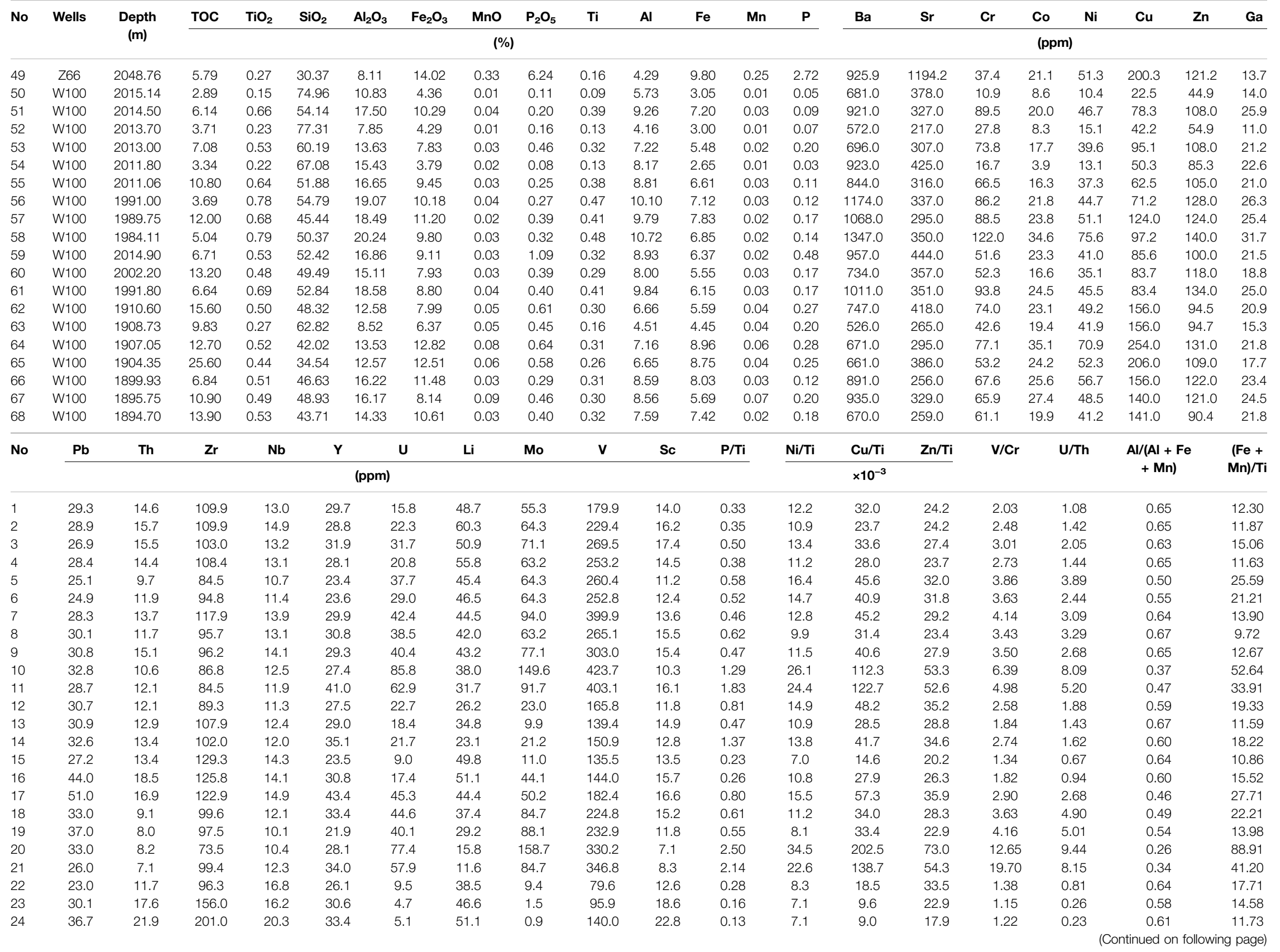


TABLE 1 | (Continued) Selected major, trace elements data and geochemical indicators of the Chang 7 black shale in Ordos Basin.

\begin{tabular}{|c|c|c|c|c|c|c|c|c|c|c|c|c|c|c|c|c|c|c|}
\hline \multirow[t]{2}{*}{ No } & $\mathrm{Pb}$ & Th & $\mathrm{Zr}$ & $\mathbf{N b}$ & $\mathbf{Y}$ & $\mathbf{U}$ & Li & Mo & $\mathbf{v}$ & Sc & $\mathrm{P} / \mathrm{Ti}$ & \multirow[t]{2}{*}{$\mathrm{Ni} / \mathrm{Ti}$} & $\mathrm{Cu} / \mathrm{Ti}$ & $\mathrm{Zn} / \mathrm{Ti}$ & \multirow[t]{2}{*}{$\mathrm{V} / \mathrm{Cr}$} & \multirow[t]{2}{*}{ U/Th } & \multirow{2}{*}{$\begin{array}{c}\mathrm{Al} /(\mathbf{A l}+\mathrm{Fe} \\
+\mathrm{Mn})\end{array}$} & \multirow{2}{*}{$\begin{array}{c}(\mathrm{Fe}+ \\
\mathrm{Mn}) / \mathrm{Ti}\end{array}$} \\
\hline & \multicolumn{11}{|c|}{ (ppm) } & & \multicolumn{2}{|l|}{$\times 10^{-3}$} & & & & \\
\hline 25 & 24.9 & 17.1 & 188.0 & 17.3 & 26.7 & 23.5 & 39.0 & 9.6 & 92.7 & 15.1 & 0.16 & 10.3 & 8.9 & 25.2 & 1.22 & 1.37 & 0.67 & 9.53 \\
\hline 26 & 33.5 & 11.1 & 105.0 & 8.8 & 34.0 & 79.5 & 38.1 & 126.0 & 238.0 & 11.9 & 0.64 & 31.8 & 114.5 & 64.5 & 5.45 & 7.16 & 0.27 & 67.23 \\
\hline 27 & 45.6 & 19.5 & 194.0 & 17.9 & 37.5 & 23.1 & 43.4 & 24.7 & 109.0 & 16.4 & 0.17 & 10.9 & 18.2 & 28.4 & 1.47 & 1.18 & 0.60 & 14.45 \\
\hline 28 & 32.8 & 15.7 & 148.0 & 13.7 & 28.6 & 41.5 & 42.9 & 68.7 & 148.0 & 13.5 & 0.46 & 20.5 & 55.8 & 57.7 & 2.48 & 2.64 & 0.51 & 34.21 \\
\hline 29 & 33.6 & 12.3 & 133.0 & 11.6 & 27.7 & 79.5 & 42.4 & 125.0 & 185.0 & 11.5 & 0.23 & 12.7 & 46.2 & 32.1 & 3.70 & 6.46 & 0.39 & 27.35 \\
\hline 30 & 26.4 & 8.3 & 82.4 & 7.9 & 20.0 & 125.0 & 24.2 & 196.0 & 304.0 & 9.1 & 0.39 & 18.7 & 87.2 & 43.0 & 6.06 & 15.10 & 0.26 & 48.10 \\
\hline 31 & 24.2 & 6.5 & 69.7 & 6.9 & 15.5 & 82.3 & 22.5 & 158.0 & 293.0 & 7.2 & 0.35 & 13.3 & 61.5 & 36.0 & 10.00 & 12.66 & 0.26 & 40.36 \\
\hline 32 & 28.8 & 9.0 & 76.0 & 7.0 & 18.3 & 59.4 & 18.9 & 110.0 & 226.0 & 8.1 & 0.70 & 22.7 & 99.5 & 52.2 & 5.89 & 6.60 & 0.32 & 58.09 \\
\hline 33 & 18.6 & 6.0 & 69.9 & 5.9 & 13.6 & 54.6 & 19.4 & 145.0 & 253.0 & 6.3 & 0.72 & 18.7 & 91.7 & 50.6 & 9.04 & 9.07 & 0.24 & 67.43 \\
\hline 34 & 26.0 & 9.5 & 132.0 & 10.0 & 16.0 & 23.6 & 15.5 & 56.0 & 133.0 & 10.6 & 0.23 & 7.8 & 22.9 & 28.5 & 3.41 & 2.48 & 0.55 & 20.13 \\
\hline 35 & 25.6 & 9.3 & 97.8 & 8.4 & 24.3 & 75.9 & 23.9 & 161.0 & 313.0 & 11.8 & 0.90 & 14.5 & 66.7 & 40.8 & 7.03 & 8.21 & 0.32 & 36.30 \\
\hline 36 & 30.6 & 10.1 & 109.0 & 10.5 & 32.5 & 86.4 & 25.4 & 139.0 & 290.0 & 15.8 & 0.71 & 15.5 & 61.5 & 38.6 & 5.88 & 8.55 & 0.36 & 36.46 \\
\hline 37 & 27.9 & 10.6 & 144.0 & 10.4 & 16.2 & 33.5 & 17.5 & 55.7 & 125.0 & 9.6 & 0.27 & 9.0 & 29.0 & 32.7 & 2.75 & 3.16 & 0.58 & 19.39 \\
\hline 38 & 24.7 & 10.3 & 119.0 & 9.4 & 18.8 & 57.6 & 25.2 & 113.0 & 179.0 & 12.1 & 0.43 & 11.9 & 46.0 & 35.2 & 2.92 & 5.59 & 0.44 & 29.23 \\
\hline 39 & 29.4 & 13.5 & 142.0 & 12.5 & 23.9 & 44.1 & 23.7 & 98.3 & 199.0 & 14.0 & 0.33 & 11.4 & 39.4 & 35.4 & 3.49 & 3.27 & 0.49 & 27.97 \\
\hline 40 & 23.7 & 9.6 & 128.0 & 10.9 & 23.0 & 29.3 & 22.2 & 29.7 & 188.0 & 12.2 & 0.63 & 13.4 & 47.4 & 31.3 & 3.53 & 3.04 & 0.40 & 31.74 \\
\hline 41 & 24.1 & 7.5 & 70.2 & 10.5 & 24.0 & 79.3 & 12.7 & 189.6 & 438.4 & 12.5 & 1.68 & 25.5 & 201.3 & 61.0 & 10.99 & 10.57 & 0.32 & 55.96 \\
\hline 42 & 31.0 & 14.6 & 86.6 & 9.4 & 32.1 & 17.2 & 11.2 & 25.4 & 92.8 & 9.6 & 0.62 & 12.5 & 237.3 & 39.8 & 3.12 & 1.18 & 0.63 & 17.29 \\
\hline 43 & 23.8 & 8.6 & 94.5 & 9.9 & 27.9 & 62.1 & 8.3 & 149.5 & 343.0 & 12.6 & 1.11 & 18.2 & 123.5 & 46.9 & 18.74 & 7.22 & 0.37 & 39.20 \\
\hline 44 & 26.2 & 9.7 & 91.2 & 10.1 & 22.0 & 23.0 & 18.6 & 38.7 & 161.7 & 11.6 & 1.52 & 15.7 & 159.7 & 44.0 & 5.50 & 2.38 & 0.39 & 40.94 \\
\hline 45 & 41.8 & 20.8 & 134.3 & 14.0 & 28.3 & 5.4 & 22.5 & 2.0 & 67.6 & 10.6 & 0.14 & 4.6 & 155.1 & 28.5 & 1.23 & 0.26 & 0.78 & 7.24 \\
\hline 46 & 28.9 & 9.3 & 78.6 & 7.7 & 20.6 & 62.7 & 26.7 & 156.0 & 354.0 & 8.1 & 1.23 & 22.3 & 103.9 & 56.6 & 8.81 & 6.74 & 0.27 & 70.23 \\
\hline 47 & 34.2 & 11.0 & 88.2 & 9.1 & 22.6 & 53.6 & 29.9 & 144.0 & 277.0 & 11.6 & 1.15 & 21.0 & 89.5 & 46.5 & 4.25 & 4.87 & 0.38 & 44.23 \\
\hline 48 & 28.0 & 9.4 & 98.4 & 12.3 & 38.2 & 86.0 & 13.0 & 99.0 & 313.9 & 9.1 & 2.57 & 33.7 & 149.4 & 78.8 & 8.87 & 9.15 & 0.26 & 89.60 \\
\hline 49 & 33.3 & 8.2 & 93.8 & 8.2 & 47.8 & 83.9 & 18.8 & 141.2 & 381.2 & 13.8 & 16.62 & 31.3 & 122.3 & 74.0 & 10.19 & 10.23 & 0.30 & 61.38 \\
\hline 50 & 76.1 & 35.8 & 91.0 & 19.6 & 26.2 & 18.5 & 15.2 & 8.2 & 15.7 & 5.4 & 0.50 & 11.3 & 24.4 & 48.6 & 1.44 & 0.52 & 0.65 & 33.08 \\
\hline 51 & 185.0 & 17.4 & 144.0 & 15.3 & 32.2 & 9.4 & 44.8 & 5.9 & 132.0 & 20.0 & 0.22 & 11.9 & 19.9 & 27.5 & 1.47 & 0.54 & 0.56 & 18.41 \\
\hline 52 & 56.7 & 13.7 & 63.7 & 15.4 & 30.3 & 15.0 & 24.1 & 11.9 & 147.0 & 5.9 & 0.51 & 11.2 & 31.3 & 40.7 & 5.29 & 1.09 & 0.58 & 22.31 \\
\hline 53 & 138.0 & 12.1 & 96.8 & 12.1 & 40.8 & 8.4 & 29.6 & 7.3 & 95.5 & 16.4 & 0.63 & 12.5 & 30.0 & 34.1 & 1.29 & 0.69 & 0.57 & 17.36 \\
\hline 54 & 112.0 & 26.1 & 177.0 & 17.9 & 35.2 & 7.5 & 14.8 & 5.2 & 28.1 & 7.6 & 0.26 & 10.1 & 38.8 & 65.9 & 1.68 & 0.29 & 0.75 & 20.57 \\
\hline 55 & 150.0 & 13.2 & 99.9 & 12.6 & 20.3 & 6.1 & 35.4 & 4.0 & 85.6 & 13.9 & 0.28 & 9.8 & 16.3 & 27.5 & 1.29 & 0.46 & 0.57 & 17.34 \\
\hline 56 & 159.0 & 11.3 & 120.0 & 14.7 & 27.1 & 6.0 & 44.5 & 3.4 & 141.0 & 18.7 & 0.25 & 9.6 & 15.2 & 27.4 & 1.64 & 0.53 & 0.59 & 15.31 \\
\hline 57 & 140.0 & 12.4 & 120.0 & 13.7 & 30.9 & 17.3 & 67.0 & 8.5 & 197.0 & 19.5 & 0.42 & 12.6 & 30.6 & 30.6 & 2.23 & 1.40 & 0.56 & 19.34 \\
\hline 58 & 183.0 & 14.9 & 174.0 & 17.8 & 47.9 & 8.7 & 57.5 & 4.5 & 182.0 & 23.0 & 0.29 & 15.9 & 20.4 & 29.4 & 1.49 & 0.58 & 0.61 & 14.46 \\
\hline 59 & 107.0 & 11.3 & 124.0 & 11.4 & 32.5 & 12.9 & 23.8 & 6.9 & 65.5 & 14.5 & 1.49 & 12.9 & 26.8 & 31.4 & 1.27 & 1.14 & 0.58 & 20.04 \\
\hline 60 & 113.0 & 13.5 & 109.0 & 11.3 & 24.0 & 12.9 & 25.5 & 9.8 & 87.9 & 12.9 & 0.59 & 12.2 & 29.0 & 40.9 & 1.68 & 0.96 & 0.59 & 19.32 \\
\hline 61 & 142.0 & 11.5 & 120.0 & 12.0 & 33.9 & 9.3 & 30.9 & 3.9 & 54.1 & 17.6 & 0.42 & 11.0 & 20.2 & 32.5 & 0.58 & 0.81 & 0.61 & 14.99 \\
\hline 62 & 100.0 & 10.0 & 194.0 & 15.7 & 37.3 & 20.7 & 28.0 & 18.6 & 187.0 & 13.4 & 0.89 & 16.4 & 52.0 & 31.5 & 2.53 & 2.07 & 0.54 & 18.77 \\
\hline 63 & 170.0 & 8.5 & 86.6 & 17.9 & 41.0 & 16.7 & 15.6 & 34.2 & 196.0 & 9.9 & 1.21 & 25.8 & 96.0 & 58.3 & 4.60 & 1.97 & 0.50 & 27.64 \\
\hline 64 & 186.0 & 12.1 & 140.0 & 19.7 & 57.6 & 30.0 & 26.7 & 107.0 & 310.0 & 16.5 & 0.90 & 22.9 & 82.1 & 42.3 & 4.02 & 2.48 & 0.44 & 29.17 \\
\hline 65 & 94.1 & 8.7 & 71.7 & 7.3 & 24.8 & 31.9 & 53.5 & 87.1 & 308.0 & 12.3 & 0.96 & 19.7 & 77.7 & 41.1 & 5.79 & 3.68 & 0.43 & 33.18 \\
\hline 66 & 131.0 & 10.6 & 84.9 & 9.4 & 22.7 & 13.1 & 52.6 & 52.5 & 154.0 & 13.1 & 0.41 & 18.4 & 50.6 & 39.6 & 2.28 & 1.24 & 0.52 & 26.13 \\
\hline 67 & 130.0 & 10.6 & 82.2 & 9.8 & 22.4 & 14.0 & 47.4 & 39.4 & 154.0 & 13.8 & 0.68 & 16.4 & 47.3 & 40.9 & 2.34 & 1.32 & 0.60 & 19.45 \\
\hline 68 & 67.3 & 9.4 & 138.0 & 16.6 & 31.8 & 12.0 & 42.5 & 36.7 & 160.0 & 11.1 & 0.55 & 13.0 & 44.5 & 28.6 & 2.62 & 1.28 & 0.50 & 23.51 \\
\hline
\end{tabular}


shale (PAAS), showing relatively high abundances of $\mathrm{Cu}, \mathrm{Pb}$, $\mathrm{Zn}$, U, and Mo (Figure 6), with mean values of 149.8, 57.6, 101.6, 36.2, and $65.6 \mathrm{ppm}$, respectively.

\section{DISCUSSION}

\section{Geological Conditions of Hydrothermal Activity}

The occurrence of hydrothermal activity often requires specific geological conditions. There are two preconditions for hydrothermal activity during the Late Triassic Chang 7 sedimentary period, including basement faults and strong tectonic activity. Previous study found that basement faults were widely distributed in the edge and middle of the Ordos Basin (Figure 2) (Qiu, 2011), providing good channels for the upwelling of deep hydrothermal fluids. In addition, strong tectonic activity is supported by the presence of structural deformations, vertical or high angle fractures, seismites, and tuff intervals during the Chang 7 sedimentary period (Xia et al., 2007b; Li et al., 2008; Xia and Tian, 2007a; Zhang et al., 2009; Chen et al., 2011; Qiu, 2011; Yang and Deng, 2013; Yuan et al., 2019), providing kinetic conditions for the activation of basement faults and the invasion of hydrothermal fluids into the bottom of the basin.

\section{Evidences of Hydrothermal Activity Mineral Petrology}

Through the observation of cores and thin-sections, many minerals indicated that lake-bottom hydrothermal activities have been found in the Chang 7 black shale. Below we overview evidence from pyrite and carbonate veins, marcasite, and gypsum.

1) Pyrite veins. In general, sedimentary pyrite is characterized by small particle size, subhedral-euhedral, or framboidal crystal. However, hydrothermal pyrite is massive, porphyritic, or veinlike (Wang et al., 2014). Pyrite veins from the Chang 7 black shale of the Yanchang Formation in this study were characterized as heterogeneous and amorphous, containing a lot of impurities (Figures 4A-D). This suggests that they may be formed by hydrothermal mineralization instead of sedimentation, strongly indicating the occurrence of hydrothermal activity.

2) Carbonate veins or laminae. In the Chang 7 core samples, many carbonate veins or laminae were found in contact with the surrounding rock at a nearly horizontal or very low angle (Figures 4E-H). The main body of a carbonate vein in Figure $\mathbf{4 H}$ (the white part) embedded in black shale was relatively thick $(\sim 1 \mathrm{~cm})$ and horizontally distributed. However, those relatively small branches were separated at its edge and intruded into the shale at a low angle, indicating that they may be formed by the intrusion of lake-bottom hydrothermal fluids into black shale before consolidation and diagenesis.

3) Marcasite. The chemical composition of marcasite is the same as that of pyrite $\left(\mathrm{FeS}_{2}\right)$. Its crystal is generally plate-shaped or sheet-shaped, and aggregated in the shape of a chicken crown or bundle (Figure 4I). Marcasite is an unstable variety of $\mathrm{FeS}_{2}$, which will transform into pyrite when temperatures are $>350^{\circ} \mathrm{C}$. The occurrence of marcasite usually indicates a relatively low temperature hydrothermal fluid (Zhang et al., 2010; Qiu, 2011).

4) Gypsum. Gypsum generally forms by evaporation or hydrothermal deposition. The Chang 7 sedimentary period was characterized by fresh lake water and a warm-humid climate (Ji and Meng, 2006; Fu et al., 2009), which is not conducive to the formation of evaporating gypsum. The gypsum crystals in the Chang 7 shale were mostly strip or block, and the crystal aggregates are distributed in belts (Figure 4J). These crystal structure characteristics and distribution indicate that gypsum in the Chang 7 Member of the Yanchang formation is a typical product of hydrothermal sedimentation (Li et al., 2016).

5) Other minerals. In addition to those listed above, many other hydrothermal minerals have been found in the Chang 7 black shale, such as siliceous rock (Qiu, 2011), manganese nodules (Qiu, 2011), authigenic albites (Zhang et al., 2010), and independent uranium deposits (Zhang et al., 2011).

There may be other explanations for the above minerals, but the widespread occurrence of various minerals in the Chang 7 Member undoubtedly supports the occurrence of hydrothermal activity.

\section{Isotope}

The carbon and oxygen isotope values of carbonate laminae in Figure 4E were $-8.2 \%$ and $-20.0 \%$, respectively, showing an obvious negative shift (Table 2). However, the $\delta^{13} \mathrm{C}$ and $\delta^{18} \mathrm{O}$ values of bulk carbonates in the Chang 7 black shale were relatively high, ranging from -1.4 to $1.6 \%$ o (mean $=0.8 \%$ ) and from $-11.1 \%$ o to $-7.5 \%$ (mean $=-9.4 \%$ ), respectively (Table 2), showing distinct differences compared to that of carbonate laminae. It is consistent with previous report of the $\delta^{13} \mathrm{C}$ and $\delta^{18} \mathrm{O}$ values in hydrothermal carbonates in Ordovician of the Ordos Basin, which were also very negative (Wang et al., 2013). This phenomenon may be caused by the large amount of heavy carbon and oxygen isotope loss in high temperature environments. Therefore, low $\delta^{13} \mathrm{C}$ and $\delta^{18} \mathrm{O}$ values of carbonate laminae also indicate that hydrothermal fluids may be involved in their formation.

Zhang et al. (2010) found that the $\delta^{34}$ S of pyrite in the Chang 7 black shale varied from 2.4 to $5.9 \%$, showing positive delta values compared with that of pyrite (mean $=-8.4 \%$ ) in the overlying Yan'an Formation. They proposed that the positive $\delta^{34} S$ in the Chang 7 shale reflected that the sulfur in pyrites may be primarily from deep hydrothermal fluids. Therefore, the $\delta^{34} S$ may also support the presence of hydrothermal activity.

\section{Major and Trace Elements}

The crossplot of the Chang 7 black shale (Figure 7) shows that most black shale samples demonstrate a positive correlation between $\mathrm{SiO}_{2}$ and $\mathrm{TiO}_{2}$, indicating that they are both almost entirely supplied by terrigenous clastics (Qiu et al., 2015). 


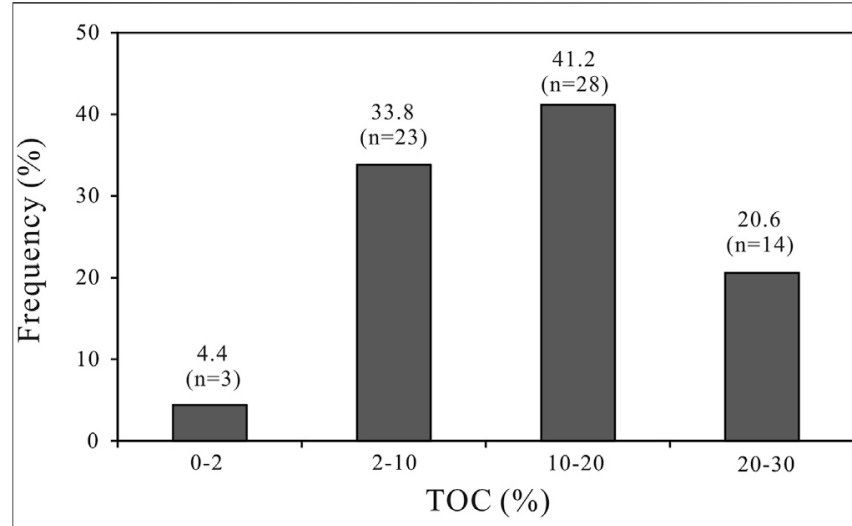

FIGURE 5|Frequency distribution of TOC contents in the Chang 7 black shale of Yanchang Formation, Ordos Basin.

TABLE 2 | Carbon and oxygen isotopes of carbonate from the Chang 7 black shale of the Upper Trassic Yanchang Formation in Ordos Basin.

\begin{tabular}{llclcc}
\hline No & Wells & Depth $(\mathbf{m})$ & Lithology & $\begin{array}{c}\boldsymbol{\delta}^{\mathbf{1 3}} \mathbf{C}(\%), \\
\mathbf{P D B})\end{array}$ & $\begin{array}{c}\boldsymbol{\delta}^{\mathbf{1 8}} \mathbf{O}(\mathbf{\%}, \\
\mathbf{P D B})\end{array}$ \\
\hline 1 & Z10 & 1421.32 & black shale & 1.3 & -10.2 \\
2 & Z119 & 2186.80 & black shale & 0.2 & -7.5 \\
3 & Z338 & 2022.63 & black shale & 0.8 & -8.1 \\
4 & G91 & 2671.20 & black shale & -1.4 & -10.5 \\
5 & N76 & 1789.30 & black shale & 1.5 & -9.3 \\
6 & Y423 & 2335.68 & black shale & 1.5 & -11.1 \\
7 & H79 & 2731.10 & black shale & 0.8 & -7.7 \\
8 & L68 & 1984.70 & black shale & 1.3 & -9.1 \\
9 & Z82 & 2011.80 & black shale & 1.6 & -9.4 \\
10 & Z82 & 2008.29 & black shale & 0.5 & -10.8 \\
11 & W100 & 1907.05 & carbonate vein & -8.2 & -20.0 \\
\hline
\end{tabular}

However, some samples (in the elliptical frame) do not conform to this rule, with abnormally high $\mathrm{SiO}_{2}$ contents (Figure 7), indicating that the $\mathrm{SiO}_{2}$ are not completely provided by terrigenous clasts. Surveys of modern submarine hydrothermal activity found that the content of $\mathrm{SiO}_{2}$ in the hydrothermal fluid is very high, generally $9.3-21.9 \mathrm{mmol} / \mathrm{kg}$, and that amorphous $\mathrm{SiO}_{2}$ tends to precipitate in hydrothermal fields (Sun et al., 2003). In addition, siliceous rocks related to hydrothermal activity have also been found in the Chang 7 Member (Qiu, 2011). Therefore, the lake-bottom hydrothermal fluid may be one of the most likely sources of abnormally high $\mathrm{SiO}_{2}$ in the study area.

$\mathrm{Al}$ and $\mathrm{Ti}$ are good indicators of terrigenous input, while $\mathrm{Fe}$ and $\mathrm{Mn}$ are usually enriched in hydrothermal fluids, suggesting their patterns can be used to distinguish the hydrothermal deposition in sediments (He et al., 2016). The Al-Fe-Mn triangular diagram of the Chang 7 black shale shows that contents of $\mathrm{Mn}(<5.0 \%)$ were far less than that of $\mathrm{Fe}$ and $\mathrm{Al}$, but the Fe contents of some samples were very rich (Figure 8A). Figure 8A also shows that only part of samples fell into the area of hydrothermal deposition, indicating that these samples may be affected by hydrothermal activity.

The values of $\mathrm{Al} /(\mathrm{Al}+\mathrm{Fe}+\mathrm{Mn})$ and $(\mathrm{Fe}+\mathrm{Mn}) / \mathrm{Ti}$ in sediments or rocks have also been successfully applied to the identification of hydrothermal activity (Qi et al., 2004; Li et al., 2014; He et al., 2016). $\mathrm{Al} /(\mathrm{Al}+\mathrm{Fe}+\mathrm{Mn})$ values $<0.4$ and $(\mathrm{Fe}+$ $\mathrm{Mn} / \mathrm{Ti}$ values $>15$ usually indicate typical hydrothermal deposition (He et al., 2016). Moreover, the value of $\mathrm{Al} /(\mathrm{Al}+$ $\mathrm{Fe}+\mathrm{Mn}$ ) is also an important proxy to evaluate the proportion of hydrothermal sedimentary components in sediments. The smaller the value is, the more hydrothermal sedimentary components are present, indicating a stronger influence of hydrothermal activity. On the contrary, a high value of $\mathrm{Al} /(\mathrm{Al}$ $+\mathrm{Fe}+\mathrm{Mn}$ ) reflects weak influence of hydrothermal activity (He

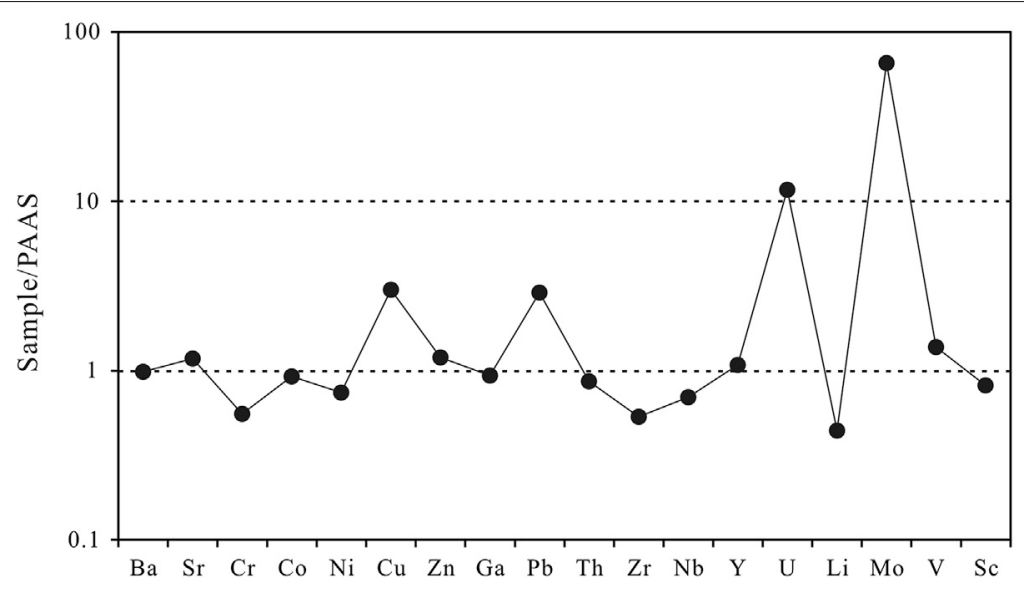

FIGURE 6 | PAAS standardization of average trace element contents of the Chang 7 black shale of Yanchang Formation, Ordos Basin. The data of PAAS are from Taylor and McLennan (1985). 


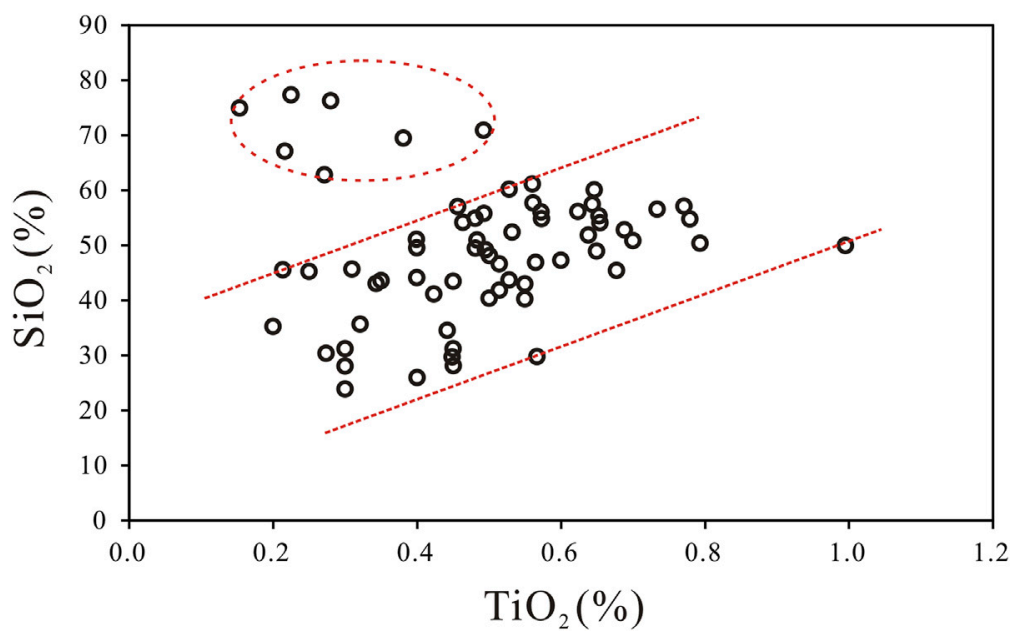

FIGURE 7 | The crossplot of $\mathrm{SiO}_{2}$ and $\mathrm{TiO}_{2}$ in the Chang 7 black shale, Ordos Basin.
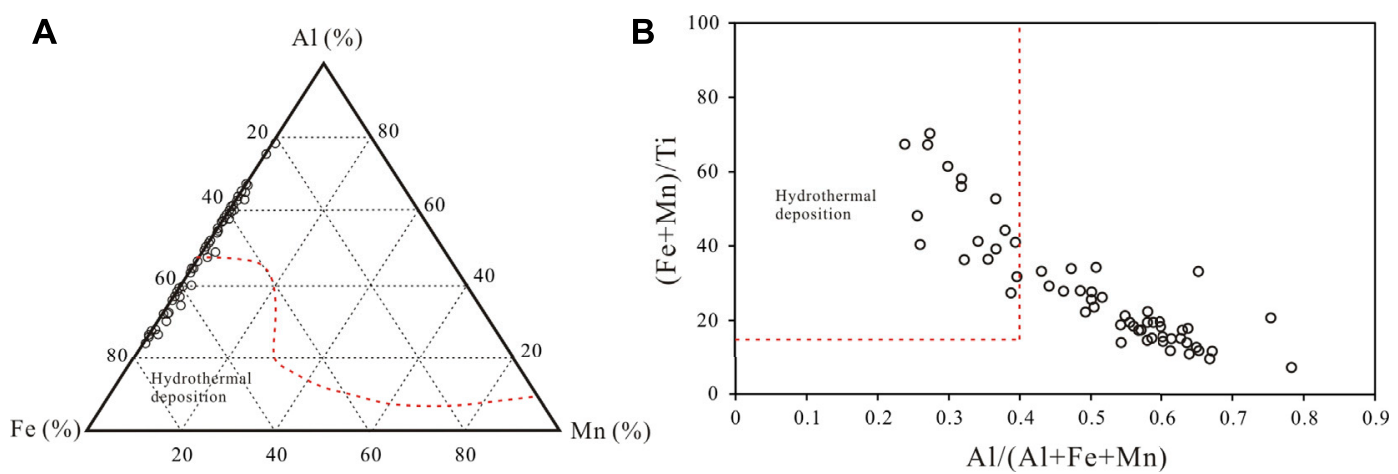

FIGURE 8 | Discrimination diagrams of hydrothermal activity based on Al, Fe, and Mn elements. Base maps are after He et al. (2016).

et al., 2016). Values of $\mathrm{Al} /(\mathrm{Al}+\mathrm{Fe}+\mathrm{Mn})$ of the Chang 7 black shale in this study ranged from 0.24 to 0.78 , with an average of 0.51 , while values of $(\mathrm{Fe}+\mathrm{Mn}) / \mathrm{Ti}$ ranged from 7.24 to 89.60 , with an average of 28.51 (Table 1). The crossplot of $\mathrm{Al} /(\mathrm{Al}+\mathrm{Fe}+\mathrm{Mn})$ and $(\mathrm{Fe}+\mathrm{Mn}) / \mathrm{Ti}$ showed similar results to that of $\mathrm{Al}-\mathrm{Fe}-\mathrm{Mn}$ triangular diagram, indicating that part of shale samples fell into the area of typical hydrothermal sedimentation (Figure 8B).

Concentrations of $\mathrm{Cu}, \mathrm{Pb}$, and $\mathrm{Zn}$ elements are related to deep hydrothermal fluids (Sun et al., 2003) and will be enriched if hydrothermal sedimentation occurs. Average concentrations of $\mathrm{Cu}, \mathrm{Pb}$, and $\mathrm{Zn}$ in the Chang 7 black shale were 149.8, 57.6, and 101.6 ppm, respectively (Table 1), showing distinct enrichment compared to the PAAS (Figure 6). From this, we can infer that deep hydrothermal activity may have occurred during the deposition of the Chang 7 black shale in the Ordos Basin.

In summary, the occurrence of lake-bottom hydrothermal activity during the Chang 7 sedimentary period is supported by evidence from mineral petrology, isotopics, major and trace elements in the Chang 7 black shale; however, its influence may only be concentrated in partial areas of the Ordos Basin.

\section{Relationship Between Hydrothermal Activity and Organic Matter Enrichment}

Most the modern hydrothermal activity occurs at the bottom of the ocean, mainly distributed near mid-ocean-ridges, plate edges, and submarine volcanic craters, while there are relatively few cases of hydrothermal activity found in terrestrial lakes (Li et al., 2014). Several biological communities have been found around the submarine or lake-bottom hydrothermal fields (Karl et al., 1980; Halbach et al., 2001; Zhang et al., 2010). Therefore, hydrothermal activity is likely of great significance to the prosperity of organisms.

The enrichment of organic matter is mainly controlled by the supply and preservation of organic matter (Arthur and Sageman, 1994; Sun, 2013; Ding, 2014; Wang et al., 2017). The former is closely related to primary productivity, while the latter is mainly related to redox conditions and sedimentation rate (Demaison and Moore, 1980; Pedersen and Calvert, 1990; Calvert and Pedersen, 1993; Curiale and Gibling, 1994; Jones and Manning, 1994; Hay, 1995; Parrish, 1995; Algeo et al., 2011; Ding et al., 2015; Wang et al., 2017; Yuan et al., 2020). Previous 

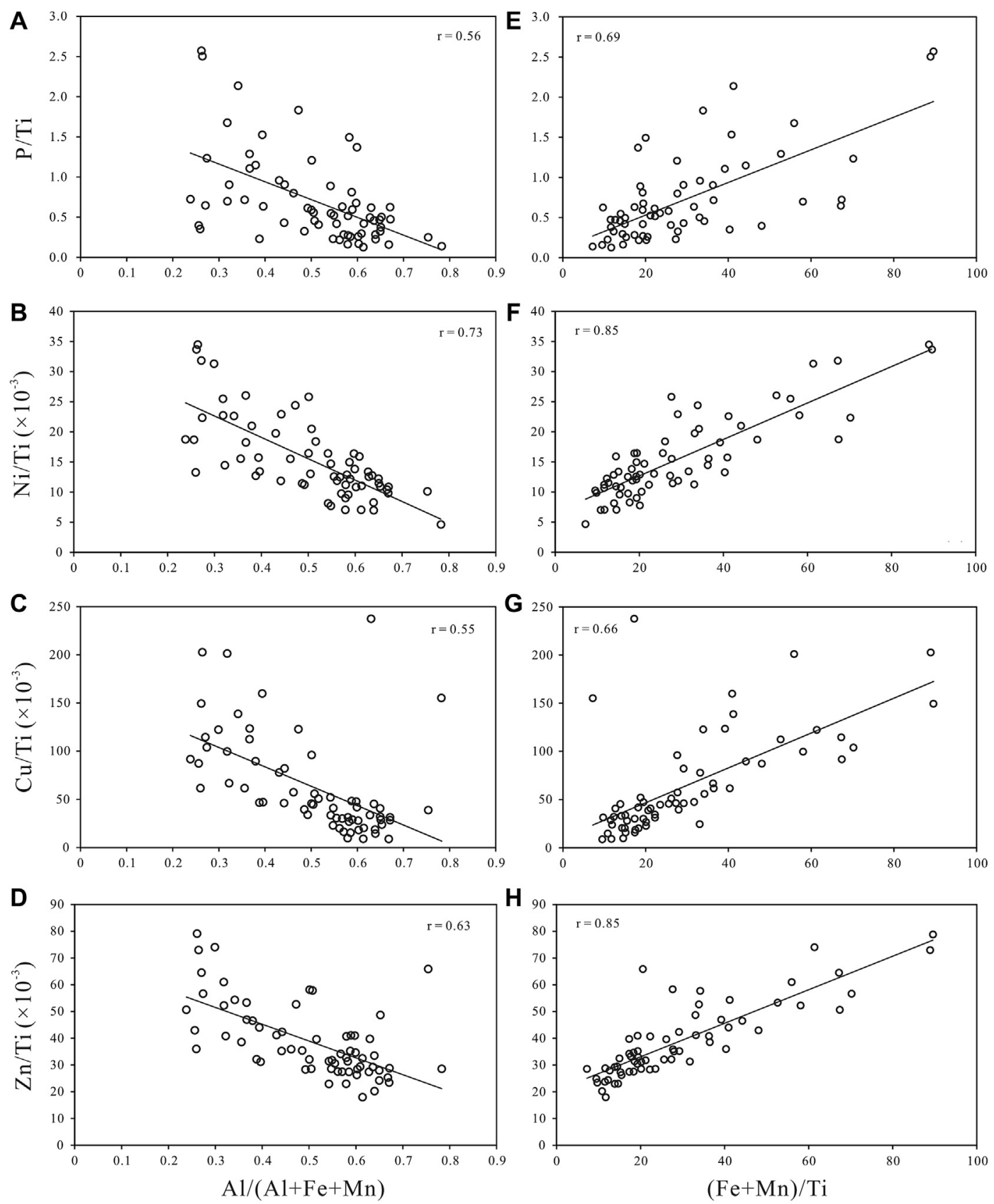

FIGURE 9 | Crossplots of paleoproductivity indicators (P/Ti, Ni/Ti, Cu/Ti, and Zn/Ti) vs. Al/(Al + Fe $+\mathrm{Mn})$ and (Fe $+\mathrm{Mn}) / \mathrm{Ti}$ of the Chang 7 black shale in the Ordos Basin.

studies have found that the sedimentation rate of the Chang 7 Member in the Ordos Basin has little effect on the enrichment of organic matter (Yuan et al., 2016, 2020). Therefore, the enrichment of organic matter in the Chang 7 shale is mainly related to paleoproductivity and redox conditions.

$\mathrm{P}, \mathrm{Ni}, \mathrm{Cu}$, and $\mathrm{Zn}$, which have been successfully used to elevate the primary paleoproductivity, are critical nutrient elements
(Algeo and Maynard, 2004; Tribovillard et al., 2006; Algeo et al., 2011; Wang et al., 2017; Yuan et al., 2020). To eliminate the influence of terrigenous clastics, palaeoproductivity indicators $(\mathrm{P}, \mathrm{Ni}, \mathrm{Cu}$, and $\mathrm{Zn})$ were normalized to $\mathrm{Ti}$, which is largely supplied in association with terrigenous minerals (Arthur and Dean, 1991; Calvert and Pedersen, 2007; Qiu et al., 2015; Wang et al., 2017; Yuan 

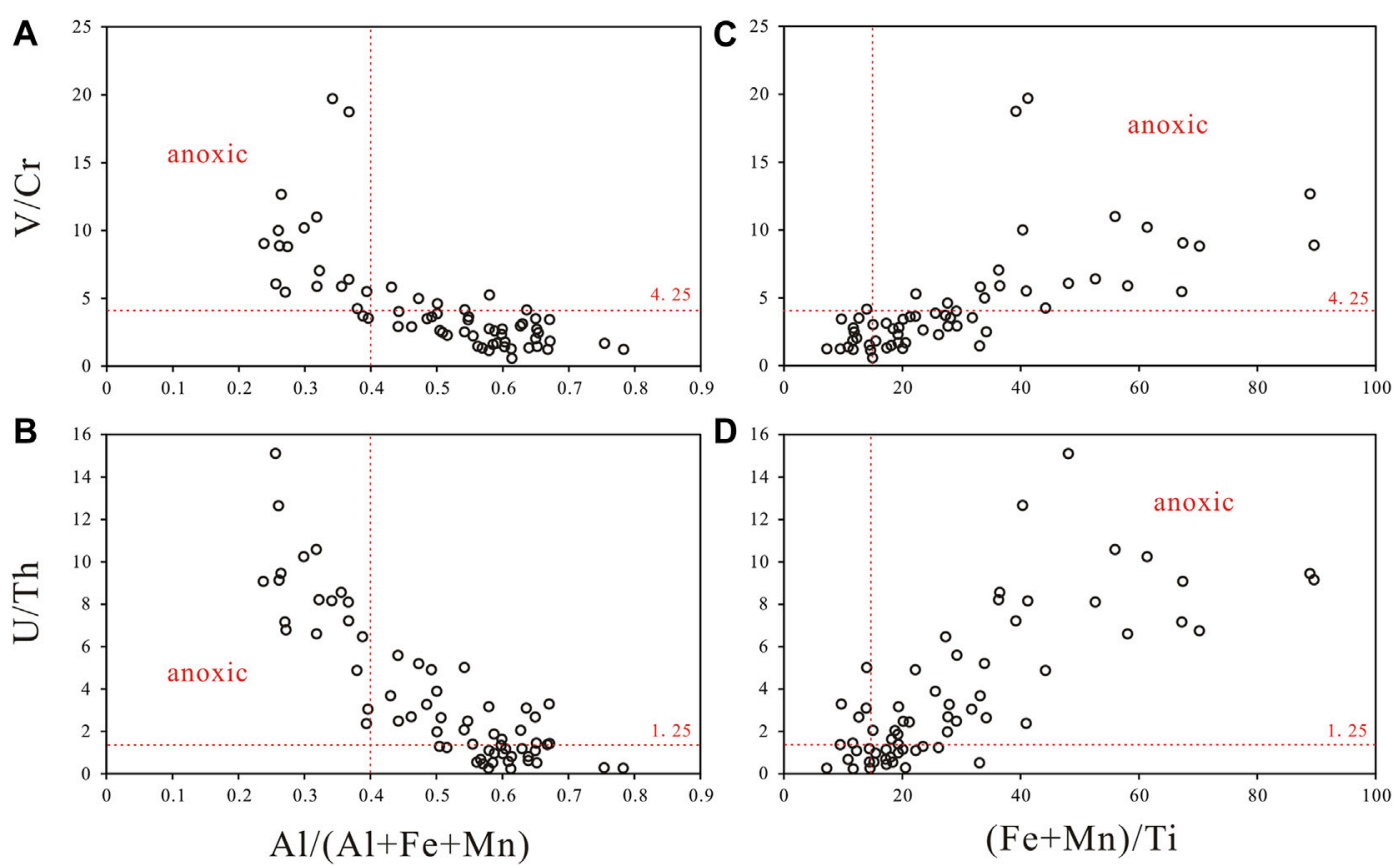

FIGURE 10 | Crossplots of V/Cr and U/Th vs. Al/(Al $+\mathrm{Fe}+\mathrm{Mn})$ and $(\mathrm{Fe}+\mathrm{Mn}) / \mathrm{Ti}$ of the Chang 7 black shale in the Ordos Basin. The threshold values of anoxic are from Jones and Manning (1994).
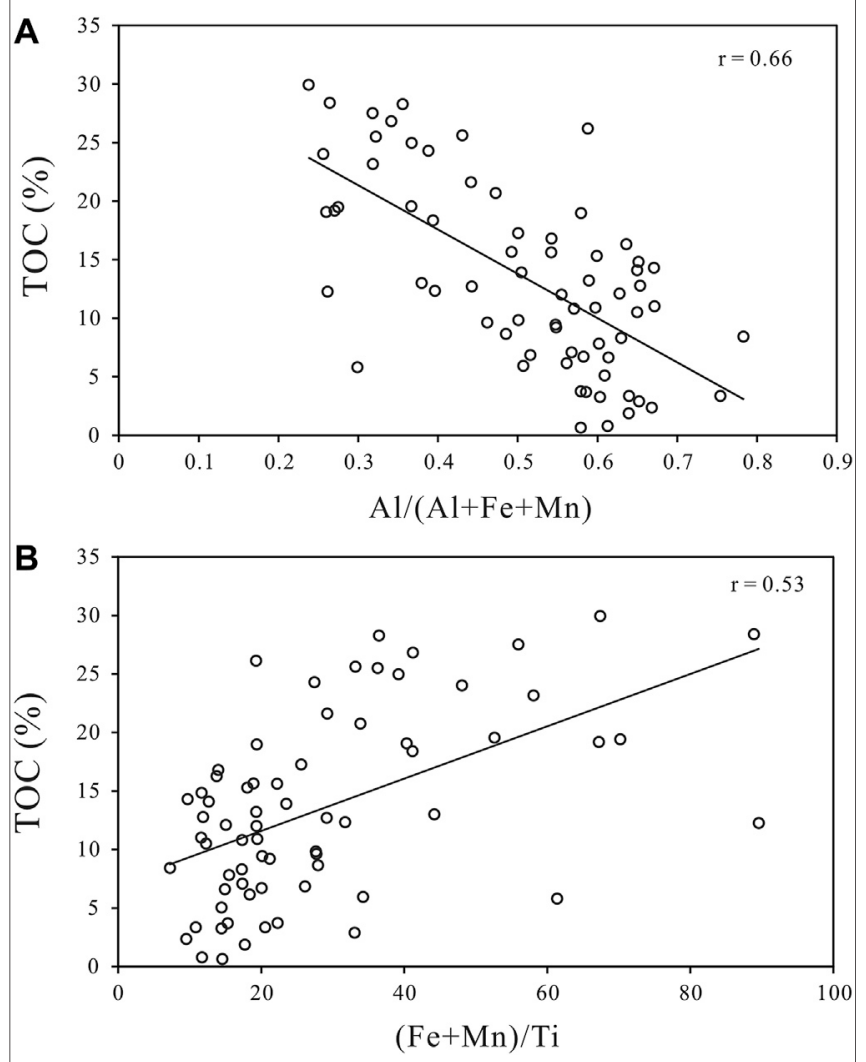

FIGURE 11 | Relationship of TOC contents with $\mathrm{Al} /(\mathrm{Al}+\mathrm{Fe}+\mathrm{Mn})$ and (Fe $+\mathrm{Mn}) / \mathrm{Ti}$ in the Chang 7 black shale of the Ordos Basin. et al., 2020). The palaeoproductivity indicators (P/Ti, Ni/Ti, Cu/ $\mathrm{Ti}$, and $\mathrm{Zn} / \mathrm{Ti}$ ) of the Chang 7 black shale samples showed strong correlations with hydrothermal indicators, including $\mathrm{Al} /(\mathrm{Al}+\mathrm{Fe}+\mathrm{Mn})$ and $(\mathrm{Fe}+\mathrm{Mn}) / \mathrm{Ti}$ (Figure 9). High values of palaeoproductivity indicators reflected high primary productivity co-occurred in areas of hydrothermal sedimentation, which is characterized by low values of $\mathrm{Al} /$ $(\mathrm{Al}+\mathrm{Fe}+\mathrm{Mn})(<0.4)$ and high values of $(\mathrm{Fe}+\mathrm{Mn}) / \mathrm{Ti}$ ( $>$ 15) (Figure 9). Our results indicate that the high paleoproductivity observed during the deposition of the Chang 7 shale may be affected by lake-bottom hydrothermal fluids, which could transport abundant nutrients into the lake water and futherly promote surface productivity.

Redox conditions during the deposition of the Chang 7 shale were oxic-suboxic accompanied by intermittent anoxic environments (Yuan et al., 2017; Chen et al., 2020). The crossplots of $\mathrm{V} / \mathrm{Cr}$ and $\mathrm{U} / \mathrm{Th}$ versus $\mathrm{Al} /(\mathrm{Al}+\mathrm{Fe}+\mathrm{Mn})$ and $(\mathrm{Fe}+\mathrm{Mn}) / \mathrm{Ti}$ showed that the anoxic environments may be related to hydrothermal activity (Figure 10). As mentioned above, hydrothermal activity could increase primary productivity. This would transport large amounts of organic matter to the water column and lake-bottom, and lead to the formation of anoxic environments due the degradation of organic matter and consumption of oxygen.

TOC contents of the Chang 7 black shale showed a strong correlation with values of $\mathrm{Al} /(\mathrm{Al}+\mathrm{Fe}+\mathrm{Mn})$ and $(\mathrm{Fe}+\mathrm{Mn}) / \mathrm{Ti}$ (Figure 11), indicating that enrichment of organic matter in the Chang 7 shale may, to some extent, be influenced by 
hydrothermal activity. This process played an important role in controlling the formation of high lacustrine primary productivity and anoxic environments.

\section{CONCLUSION}

1) Basement faults in the margin and interior of the Ordos Basin along with strong tectonic activities provide the precondition for the occurrence of lake-bottom hydrothermal activity during the Chang 7 sedimentary period.

2) The existence of lake-bottom hydrothermal activity during the Chang 7 period is supported by mineral petrology, isotopes, major and trace elements in the Chang 7 black shale.

3) Hydrothermal activities transported a large amount of nutrients to the lake water and then promoted the surface primary productivity, leading to the formation of anoxic bottom-water environment to a certain extent. Hydrothermal activities controlled the accumulation of organic matter in the Chang 7 shale by affecting the primary paleoproductivity and redox environments.

\section{REFERENCES}

Adachi, M., Yamamoto, K., and Sugisaki, R. (1986). Hydrothermal Chert and Associated Siliceous Rocks from the Northern Pacific Their Geological Significance as Indication Od Ocean Ridge Activity. Sediment. Geology. 47, 125-148. doi:10.1016/0037-0738(86)90075-8

Algeo, T. J., Kuwahara, K., Sano, H., Bates, S., Lyons, T., Elswick, E., et al. (2011). Spatial Variation in Sediment Fluxes, Redox Conditions, and Productivity in the Permian-Triassic Panthalassic Ocean. Palaeogeogr. Palaeoclimatol. Palaeoecol. 308, 65-83. doi:10.1016/j.palaeo.2010.07.007

Algeo, T. J., and Maynard, J. B. (2004). Trace-element Behavior and Redox Facies in Core Shales of Upper Pennsylvanian Kansas-Type Cyclothems. Chem. Geology. 206, 289-318. doi:10.1016/j.chemgeo.2003.12.009

Arthur, M. A., and Dean, W. E. (1991). "A Holistic Geochemical Approach to Cyclomania: Examples from Cretaceous Pelagic limestone Sequences," in Cycles and Events in Stratigraphy. Editors G. Einsele, W. Ricken, and A. Seilacher (Berlin: Springer-Verlag), 126-166.

Arthur, M. A., and Sageman, B. B. (1994). Marine Black Shales: Depositional Mechanisms and Environments of Ancient Deposits. Annu. Rev. Earth Planet. Sci. 22, 499-551. doi:10.1146/annurev.ea.22.050194.002435

Boström, K., Rydell, H., and Joensuu, O. (1979). Langban - an Exhalative Sedimentary deposit. Econ. Geology. 74, 1002-1011.

Calvert, S. E., and Pedersen, T. F. (2007). "Chapter Fourteen Elemental Proxies for Palaeoclimatic and Palaeoceanographic Variability in Marine Sediments: Interpretation and Application," in Paleoceanography of the Late Cenozoic. Part 1. Methods in Ate Cenozoic Paleoceanography. Editors C. Hillaire-Marcel and A. de Vernal (New York: Elsevier), 567-644. doi:10.1016/s1572-5480(07)01019-6

Calvert, S. E., and Pedersen, T. F. (1993). Geochemistry of Recent Oxic and Anoxic marine Sediments: Implications for the Geological Record. Mar. Geology. 113, 67-88. doi:10.1016/0025-3227(93)90150-t

Chen, A. Q., Chen, H. D., Hou, M. C., Lou, Z. H., Xu, S. L., Li, J., et al. (2011). The Middle-Late Triassic Event Sediments in Ordos Basin: Indicators for Episode I of the Indosinian Movement. Acta Geologica Sinica 85 (10), 1681-1689. (In Chinese with English abstract).

\section{DATA AVAILABILITY STATEMENT}

The original contributions presented in the study are included in the article/supplementary material, further inquiries can be directed to the corresponding author.

\section{AUTHOR CONTRIBUTIONS}

WY: Conceptualization; Methodology; Software; Investigation; Writing-Original Draft. GL: Funding acquisition; Project administration; Resources; Supervision; Validation. XZ: Validation, Software, Visualization. AB: Writing-review and editing.

\section{FUNDING}

This work was supported by the National Natural Science Foundation of China (No. 41772127).

\section{ACKNOWLEDGMENTS}

We are grateful to Zhe Cao, Wenbin Luo, and Chaozheng Li for helping to collect samples.

Chen, J. F., Sun, S. L., Liu, W. H., and Zheng, J. J. (2004). Geochemical Characteristics and Genesis of Organic-Rich Layers of Lower Cambrian in Tarim Basin. Sci. China-Earth Sci. 34, 107-113. doi:10.3969/j.issn.1674-7240. 2004.z1.012

Chen, R., Liu, G., Shang, F., and Cao, Y. (2020). Variations in Hydrocarbon Generating Potential of the Chang 7 Shale: Evidence from Pyrite Morphology and Sulfur Isotope. J. Pet. Sci. Eng. 195, 107747. doi:10.1016/j.petrol.2020. 107747

Crerar, D. A., Namson, J., Chyi, M. S., Williams, L., and Feigenson, M. D. (1982). Manganiferous Cherts of the Franciscan Assemblage; I, General Geology, Ancient and Modern Analogues, and Implications for Hydrothermal Convection at Oceanic Spreading Centers. Econ. Geology. 77, 519-540. doi:10.2113/gsecongeo.77.3.519

Cronan, D. S., Varnavas, S., and Perissoratis, C. (1995). Hydrothermal Sedimentation in the Caldera of Santorini, Hellenic Volcanic Arc. Terra Nova 7, 289-293. doi:10.1111/j.1365-3121.1995.tb00696.x

Curiale, J. A., and Gibling, M. R. (1994). Productivity Control on Oil Shale Formation-Mae Sot Basin, Thailand. Org. Geochem. 21, 67-89. doi:10.1016/ 0146-6380(94)90088-4

Dekov, V., Boycheva, T., Hålenius, U., Billström, K., Kamenov, G. D., Shanks, W. C., et al. (2011). Mineralogical and Geochemical Evidence for Hydrothermal Activity at the West wall of $12^{\circ} 50^{\prime} \mathrm{N}$ Core Complex (Mid-Atlantic Ridge): A New Ultramafic-Hosted Seafloor Hydrothermal deposit. Mar. Geology. 288, 90-102. doi:10.1016/j.margeo.2011.09.002

Demaison, G. J., and Moore, G. T. (1980). Anoxic Environments and Oil Source Bed Genesis. Org. Geochem. 2, 9-31. doi:10.1016/0146-6380(80) 90017-0

Deng, X. Q., Liu, F. X., Liu, X. Y., Pang, J. L., Lv, J. W., Li, S. X., et al. (2008). Discussion on Relationship between Sedimentary Evolution of the Triassic Yanchang Formation and the Early Indosinian Movement in Ordos Basin. J. Palaeogeogr. 10, 159-166. doi:10.7605/gdlxb.2008.02.005

Dias, Á. S., and Barriga, F. J. A. S. (2006). Mineralogy and Geochemistry of Hydrothermal Sediments from the Serpentinite-Hosted Saldanha Hydrothermal Field $\left(36^{\circ} 34^{\prime} \mathrm{N} ; 33^{\circ} 26^{\prime} \mathrm{W}\right)$ at MAR. Mar. Geology. 225, 157-175. doi:10.1016/j.margeo.2005.07.013 
Ding, X. J. (2014). Formation Mechanism of Effective Source Rock and its Control on Hydrocarbon Accumulation in Small Faulted Lacustrine basin, a Case Study of Erlian Basin. Doctoral dissertation. Beijing, China: China University of Petroleum, 115 (In Chinese with English abstract).

Ding, X., Liu, G., Zha, M., Huang, Z., Gao, C., Lu, X., et al. (2015). Relationship between Total Organic Carbon Content and Sedimentation Rate in Ancient Lacustrine Sediments, a Case Study of Erlian Basin, Northern China. J. Geochemical Exploration 149, 22-29. doi:10.1016/j.gexplo.2014.11.004

Fu, Q., Sun, X. T., and Liu, Y. D. (2009). Geological Significance and ReEstablishment of basin Character in Late Triassic of Ordos Basin. J. Tongji Univ. (Natural Science) 37 (11), 1537-1540. (in Chinese with English abstract). doi:10.3969/j.issn.0253-374x.2009.11.023

Gao, P., Liu, G., Jia, C., Ding, X., Chen, Z., Dong, Y., et al. (2015). Evaluating Rare Earth Elements as a Proxy for Oil-Source Correlation. A Case Study from Aer Sag, Erlian Basin, Northern China. Org. Geochem. 87, 35-54. doi:10.1016/j. orggeochem.2015.07.004

Halbach, P., Holzbecher, E., Koschinsky, A., Michaelis, W., and Seifert, R. (2001). Deep-sea Hydrothermal Microplume Generation-A Case Study from the North Fiji Basin. Geo-Marine Lett. 21, 94-102. doi:10.1007/s003670100068

Hay, W. W. (1995). "Paleooceanography of marine Organic-Carbon-Rich Sediments," in Paleoclimate, and Source Rocks. Editor A. Y. Huc (Middlesex, United Kingdom: American Association of Petroleum Geologists Studies in Geology), 347.

He, C., Ji, L. M., Su, A., Liu, Y., Li, J. F., Wu, Y. D., et al. (2017). Relationship between Hydrothermal Sedimentation Process and Source Rock Development in the Yanchang Formation, South Ordos Basin. Earth Sci. Front. 24, 1-9. (in Chinese with English abstract). doi:10.13745/j.esf.yx.2016-11-29

He, C., Ji, L., Wu, Y., Su, A., and Zhang, M. (2016). Characteristics of Hydrothermal Sedimentation Process in the Yanchang Formation, South Ordos Basin, China: Evidence from Element Geochemistry. Sediment. Geology. 345, 33-41. doi:10. 1016/j.sedgeo.2016.09.001

Hey, R. N., Massoth, G. J., Vrijenhoek, R. C., Rona, P. A., Lupton, J., and Butterfield, D. A. (2006). Hydrothermal Vent Geology and Biology at Earth's Fastest Spreading Rates. Mar. Geophys. Res. 27, 137-153. doi:10.1007/s11001-005$1887-\mathrm{x}$

Hodkinson, R. A., and Cronan, D. S. (1995). Hydrothermal Sedimentation at ODP Sites 834 and 835 in Relation to Crustal Evolution of the Lau Backarc Basin. Geol. Soc. Lond. Spec. Publications 87, 231-248. doi:10.1144/gsl.sp.1995.087. 01.19

Ji, L., and Meng, F. (2006). Palynology of Yanchang Formation of Middle and Late Triassic in Eastern Gansu Province and its Paleoclimatic Significance. J. China Univ. Geosciences 17 (3), 209-220. doi:10.1016/s1002-0705(06)60030-7

Jia, Z. B., Hou, D. J., Sun, D. Q., and Huang, Y. X. (2016). Hydrothermal Sedimentary Discrimination Criteria and its Coupling Relationship with Source Rocks. Nat. Gas Geosci. 27 (6), 1025-1034. (In Chinese with English abstract). doi:10.11764/j.issn.1672-1926.2016.06.1025

Jones, B., and Manning, D. A. C. (1994). Comparison of Geochemical Indices Used for the Interpretation of Palaeoredox Conditions in Ancient Mudstones. Chem. Geology. 111, 111-129. doi:10.1016/0009-2541(94)90085-x

Karl, D. M., Wirsen, C. O., and Jannasch, H. W. (1980). Deep-Sea Primary Production at the Galapagos Hydrothermal Vents. Science 207, 1345-1347. doi:10.1126/science.207.4437.1345

Li, H., Zhai, M., Zhang, L., Gao, L., Yang, Z., Zhou, Y., et al. (2014). Distribution, Microfabric, and Geochemical Characteristics of Siliceous Rocks in central Orogenic Belt, China: Implications for a Hydrothermal Sedimentation Model. Scientific World J. 2014, 1-25. doi:10.1155/2014/780910

Li, J. Y., Wang, Y. S., Liu, C. H., Dong, D. W., and Gao, Z. Q. (2016). Hydrothermal Fluid Activity and the Quantitative Evaluation of its Impact on Carbonate Reservoirs: A Case Study of the Lower Paleozoic in the West of Dongying Sag, Bohai Bay Basin, East China. Pet. Exploration Develop. 43 (5), 359-366. doi:10. 1016/s1876-3804(16)30046-5

Li, Y. H., Liu, C. Y., and Wang, X. J. (2008). Discovery and Significance of Seismites in Late Triassic Yanchang Formation of Ordos Basin. Acta Sedimentologica Sinica 26 (5), 772-778. (In Chinese with English abstract). doi:10.14027/j.cnki. cjxb.2008.05.004

Liang, Y., Hou, D. J., Zhang, J. C., and Yang, G. Q. (2014b). Influences of the Hydrothermal Activities on the Organic Matter Abundance of the Shale in
Anoxic Environment. Pet. Geology. Oilfield Develop. Daqing 33, 158-165. (in Chinese with English abstract). doi:10.3969/j.issn.1009-9603.2014.04.007

Liang, Y., Hou, D. J., Zhang, J. C., and Yang, G. Q. (2014a). The Relationship between Hydrothermal Activity and Organic-Rich Source Rocks: A Case of Lower Cambrian Niutitang Formation in the Northwest of Guizhou Area. Pet. Geology. Recovery Efficiency 21, 28-32. (in Chinese with English abstract). doi:10.3969/j.issn.1000-3754.2014.04.033

Liu, B., Bechtel, A., Sachsenhofer, R. F., Gross, D., Gratzer, R., and Chen, X. (2017). Depositional Environment of Oil Shale within the Second Member of Permian Lucaogou Formation in the Santanghu Basin, Northwest China. Int. J. Coal Geology. 175, 10-25. doi:10.1016/j.coal.2017.03.011

Liu, B., He, S., Meng, L., Fu, X., Gong, L., and Wang, H. (2021a). Sealing Mechanisms in Volcanic Faulted Reservoirs in Xujiaweizi Extension, Northern Songliao Basin, Northeastern China. Bulletin 105, 1721-1743. doi:10.1306/03122119048

Liu, B., Sun, J., Zhang, Y., He, J., Fu, X., Yang, L., et al. (2021b). Reservoir Space and Enrichment Model of Shale Oil in the First Member of Cretaceous Qingshankou Formation in the Changling Sag, Southern Songliao Basin, NE China. Pet. Exploration Develop. 48, 608-624. doi:10.1016/s1876-3804(21) 60049-6

Ma, P., Wang, L., Wang, C., Wu, X., and Wei, Y. (2015). Organic-Matter Accumulation of the Lacustrine Lunpola Oil Shale, Central Tibetan Plateau: Controlled by the Paleoclimate, Provenance, and Drainage System. Int. J. Coal Geology. 147-148, 58-70. doi:10.1016/j.coal.2015.06.011

Marchig, V., Gundlach, H., Möller, P., and Schley, F. (1982). Some Geochemical Indicators for Discrimination between Diagenetic and Hydrothermal Metalliferous Sediments. Mar. Geology. 50, 241-256. doi:10.1016/00253227(82)90141-4

Parrish, J. T. (1995). "Paleogeography of $C_{\text {org }}$-Rich Rocks and the Preservation versus Production Controversy," in Paleogeography, Paleoclimate, and Source Rocks. Editor A. Y. Huc (Middlesex, United Kingdom: American Association of Petroleum Geologists Studies in Geology), 347. doi:10.1306/st40595c1

Pedersen, T. F., and Calvert, S. E. (1990). Anoxia vs. Productivity: What Controls the Formation of Organic-Carbon-Rich Sediments and Sedimentary Rocks. AAPG Bull. 74, 454-466. doi:10.1306/0c9b232b-1710-11d7-8645000102c1865d

Pichler, T., and Dix, G. R. (1996). Hydrothermal Venting within a Coral Reef Ecosystem, Ambitle Island, Papua New Guinea. Geol 24, 435-438. doi:10.1130/ 0091-7613(1996)024<0435:hvwacr >2.3.co;2

Qi, H., Hu, R., Su, W., Qi, L., and Feng, J. (2004). Continental Hydrothermal Sedimentary Siliceous Rock and Genesis of Superlarge Germanium $(\mathrm{Ge})$ deposit Hosted in Coal: a Study from the Lincang Ge deposit, Yunnan, China. Sci. China Ser. D-Earth Sci. 47, 973-984. doi:10.1360/02yc0141

Qiu, X., Liu, C., Mao, G., Deng, Y., Wang, F., and Wang, J. (2014). Late Triassic Tuff Intervals in the Ordos basin, Central China: Their Depositional, Petrographic, Geochemical Characteristics and Regional Implications. J. Asian Earth Sci. 80, 148-160. doi:10.1016/j.jseaes.2013.11.004

Qiu, X., Liu, C., Mao, G., Deng, Y., Wang, F., and Wang, J. (2015). Major, Trace and Platinum-Group Element Geochemistry of the Upper Triassic Nonmarine Hot Shales in the Ordos basin, Central China. Appl. Geochem. 53, 42-52. doi:10. 1016/j.apgeochem.2014.11.028

Qiu, X. W., 2011. Characteristics and Dynamic Settings of Yanchang Period Hydrocarbon-Rich Depression in Ordos Basin, China. Doctoral dissertation. Xi'an, China, Northwest University, 217 (In Chinese with English abstract).

Rona, P. A. (1988). Hydrothermal Mineralization at Oceanic Ridges. Can. Mineral. 26, 431-465.

Rona, P. A., Mcgregor, B. A., Betzer, P. R., Bolger, G. W., and Krause, D. C. (1975). Anomalous Water Temperatures over Mid-Atlantic Ridge Crest at $26^{\circ}$ North Latitude. Deep Sea Res. Oceanographic Abstr. 22, 611-618. doi:10.1016/00117471(75)90048-0

Slack, J. F., Selby, D., and Dumoulin, J. A. (2015). Hydrothermal, Biogenic, and Seawater Components in Metalliferous Black Shales of the Brooks Range, Alaska: Synsedimentary Metal Enrichment in a Carbonate Ramp Setting. Econ. Geology. 110, 653-675. doi:10.2113/econgeo.110.3.653

Sun, P. C. (2013). Environmental Dynamics of Organic Accumulation in the Oil Shale Bearing Layers in the Upper Cretaceous, Southeast Songliao Basin (NE China). Doctoral dissertation. Changchun, China: Jilin University, 193 (In Chinese with English abstract). 
Sun, S. L., Chen, J. F., Liu, W. H., Zhang, S. C., and Wang, D. R. (2003). Hydrothermal Venting on the Seafloor and Formation of Organic-Rich Sediments: Evidence from the Neoproterozoic Xiamaling Formation, North China. Geol. Rev. 49, 588-595. (in Chinese with English abstract). doi:10.3321/j. issn:0371-5736.2003.06.005

Tarasov, V. G., Gebruk, A. V., Mironov, A. N., and Moskalev, L. I. (2005). Deep-sea and Shallow-Water Hydrothermal Vent Communities: Two Different Phenomena. Chem. Geology. 224, 5-39. doi:10.1016/j.chemgeo. 2005.07.021

Taylor, S. R., and McLennan, S. M. (1985). The Continental Crust: Its Composition and Evolution. Oxford, London: Blackwell Scientific Publications, 312.

Tribovillard, N., Algeo, T. J., Lyons, T., and Riboulleau, A. (2006). Trace Metals as Paleoredox and Paleoproductivity Proxies: an Update. Chem. Geology. 232, 12-32. doi:10.1016/j.chemgeo.2006.02.012

Van Dever, C. L., Desbruyeres, D., Segonzac, M., Comtet, T., Saldanha, L., FinalaMedioni, A., et al. (1996). Biology of the Lucky Strike Hydrothermal Field. Deep-sea Res. 43, 1509-1529.

Wang, C. S., Yang, J. Y., Zhang, D. S., and Zhu, L. Z. (2006). A Review on Deep-Sea Hydrothermal Vent Communities. J. Xiamen Univ. (Natural Science) 45, 141-149. (in Chinese with English abstract). doi:10.3321/j.issn:0438-0479. 2006.z2.020

Wang, C., Wang, Q., Chen, G., He, L., Xu, Y., Chen, L., et al. (2017). Petrographic and Geochemical Characteristics of the Lacustrine Black Shales from the Upper Triassic Yanchang Formation of the Ordos Basin, China: Implications for the Organic Matter Accumulation. Mar. Pet. Geology. 86, 52-65. doi:10.1016/j. marpetgeo.2017.05.016

Wang, L. L., Ling, J. X., and Hu, J. H. (2008). Recent Progress in Deep-Sea Hydrothermal Vent Communities. Adv. Earth Sci. 23, 604-612. (in Chinese with English abstract). doi:10.3321/j.issn:1001-8166.2008.06.007

Wang, Q. C., Zhang, Y., and Xiao, L. (2013). Carbon and Oxygen Stable Isotopic Features of Diagenetic Facies of Ordovician Carbonate Rocks in Ordos Basin. Oil Gas Geology. 34 (5), 652-658. (in Chinese with English abstract). doi:10. $11743 /$ ogg 20130511

Wang, Y. P., Dong, C. M., Chen, H. D., Su, Z. T., Zhang, C. J., and Hao, Z. M. (2014). Petrological Evidence of Ordovician Hydrothermal Activities and its Geological Significance to Reservoir Development in central and Western Parts of Ordos Basin. Mar. Origin Pet. Geology. 19 (2), 23-31. (in Chinese with English abstract). doi:10.3969/j.issn.1672-9854.2014.02.004

Wen, H., Zheng, R., Qing, H., Fan, M., Li, Y., and Gong, B. (2013). Primary Dolostone Related to the Cretaceous Lacustrine Hydrothermal Sedimentation in Qingxi Sag, Jiuquan Basin on the Northern Tibetan Plateau. Sci. China Earth Sci. 56, 2080-2093. doi:10.1007/s11430-013-4654-x

Xia, Q. S., and Tian, J. C. (2007a). Characteristics and Geological Significance of Seismites of the Yanchang Formation, Upper Triassic, Ordos Basin. Acta Sedimentologica Sinica 25 (2), 246-252. (In Chinese with English abstract). doi:10.3969/j.issn.1000-0550.2007.02.012

Xia, Q. S., Tian, J. C., and Huang, C. W. (2007b). Study on the Recognition marks of Seismites in Upper Triassic Yanchang Formation, Ordos Basin, China. J. Chengdu Univ. Technol. (Science Technol. Edition) 34 (3), 312-316. (In Chinese with English abstract). doi:10.3969/j.issn.1671-9727.2007.03.015

Yamamoto, K. (1987). Geochemical Characteristics and Depositional Environments of Cherts and Associated Rocks in the Franciscan and Shimanto Terranes. Sediment. Geology. 52, 65-108. doi:10.1016/00370738(87)90017-0

Yang, H., and Deng, X. Q. (2013). Deposition of Yanchang Formation deepwater sandstone under the Control of Tectonic Events, Ordos Basin. Pet. Exploration Develop. 40 (5), 513-520. doi:10.1016/s1876-3804(13)60072-5

Yang, H., Zhang, W., Wu, K., Li, S., Peng, P. a., and Qin, Y. (2010). Uranium Enrichment in Lacustrine Oil Source Rocks of the Chang 7 Member of the Yanchang Formation, Erdos Basin, China. J. Asian Earth Sci. 39, 285-293. doi:10.1016/j.jseaes.2010.03.013

Yang, H., and Zhang, W. Z. (2005). Leading Effect of the Seventh Member HighQuality Source Rock of Yanchang Formation in Ordos Basin during the
Enrichment of Low-Penetrating Oil-Gas Accumulation: Geology and Geochemistry. Geochimica 34, 147-154. (in Chinese with English abstract). doi:10.3321/j.issn:0379-1726.2005.02.007

Yuan, W., Liu, G. D., and Luo, W. B. (2016). Deposition Rate of 7th Member of Yanchang Formation, Ordos Basin and its Impact on Organic Matter Abundance of Hydrocarbon Source Rock. J. Xi'an Shiyou Univ. (Natural Sci. Edition) 31 (5), 20-26. (In Chinese with English abstract). doi:10.3969/j. issn.1673-064X.2016.05.003

Yuan, W., Liu, G., Stebbins, A., Xu, L., Niu, X., Luo, W., et al. (2017). Reconstruction of Redox Conditions during Deposition of Organic-Rich Shales of the Upper Triassic Yanchang Formation, Ordos Basin, China. Palaeogeogr. Palaeoclimatol. Palaeoecol. 486, 158-170. doi:10.1016/j.palaeo. 2016.12.020

Yuan, W., Liu, G., Xu, L., Niu, X., and Li, C. (2019). Petrographic and Geochemical Characteristics of Organic-Rich Shale and Tuff of the Upper Triassic Yanchang Formation, Ordos Basin, China: Implications for Lacustrine Fertilization by Volcanic Ash. Can. J. Earth Sci. 56, 47-59. doi:10.1139/cjes-2018-0123

Yuan, W., Liu, G., Zhou, X., Xu, L., and Li, C. (2020). Palaeoproductivity and Organic Matter Accumulation during the Deposition of the Chang 7 OrganicRich Shale of the Upper Triassic Yanchang Formation, Ordos Basin, China. Geol. J. 55, 3139-3156. doi:10.1002/gj.3578

Zhang, B. H., Wu, B. L., Liu, C. Y., and Qiu, X. W. (2011). Occurrence of Uranium in Hydrocarbon of Chang 7 Member of Yanchang Formation of Ordos Basin. Northwest. Geology. 44 (2), 124-132. (in Chinese with English abstract). doi:10. 3969/j.issn.1009-6248.2011.02.014

Zhang, W., Yang, W., and Xie, L. (2017). Controls on Organic Matter Accumulation in the Triassic Chang 7 Lacustrine Shale of the Ordos Basin, central China. Int. J. Coal Geology. 183, 38-51. doi:10.1016/j.coal. 2017.09.015

Zhang, W. Z., Yang, H., Peng, P. A., Yang, Y. H., Zhang, H., and Shi, X. H. (2009). The Influence of Late Triassic Volcanism on the Development of Chang 7 High Grade Hydrocarbon Source Rock in Ordos Basin. Geochimica 38, 573-582. (in Chinese with English abstract). doi:10.3321/j.issn:0379-1726.2009.06.007

Zhang, W. Z., Yang, H., Xie, L. Q., and Yang, Y. H. (2010). Lake-Bottom Hydrothermal Activities and Their Influence on High-Quality Source Rock Development: A Case from Chang 7 Source Rocks in Ordos Basin. Pet. Exploration Develop. 37, 424-429. doi:10.1016/s1876-3804(10)60043-2

Zheng, R. C., Wen, H. G., Fan, M. T., Wang, M. F., Wu, X. G., and Xia, P. F. (2006). Lithological Characteristics of Sublacustrine white Smoke Type Exhalative Rock of the Xiagou Formation in Jiuxi Basin. Acta Petrologica Sinica 22, 3027-3038. (in Chinese with English abstract). doi:10.1007/s10114-005-0804-1

Zhong, D. K., Jiang, Z. C., Guo, Q., and Sun, H. T. (2015). A Review about Research History, Situation and Prospects of Hydrothermal Sedimentation. J. Palaeogeogr. 17, 285-296. (in Chinese with English abstract). doi:10.7605/ gdlxb.2015.03.024

Conflict of Interest: The authors declare that the research was conducted in the absence of any commercial or financial relationships that could be construed as a potential conflict of interest.

Publisher's Note: All claims expressed in this article are solely those of the authors and do not necessarily represent those of their affiliated organizations, or those of the publisher, the editors and the reviewers. Any product that may be evaluated in this article, or claim that may be made by its manufacturer, is not guaranteed or endorsed by the publisher.

Copyright (c) 2022 Yuan, Liu, Zhou and Bulseco. This is an open-access article distributed under the terms of the Creative Commons Attribution License (CC BY). The use, distribution or reproduction in other forums is permitted, provided the original author(s) and the copyright owner(s) are credited and that the original publication in this journal is cited, in accordance with accepted academic practice. No use, distribution or reproduction is permitted which does not comply with these terms. 